KCL-MTH-07-04

\title{
The $E_{11}$ origin of all maximal supergravities
}

\author{
Fabio Riccioni and Peter West \\ Department of Mathematics \\ King's College London \\ Strand London WC2R 2LS \\ $U K$
}

\begin{abstract}
Starting from the eleven dimensional $E_{11}$ non-linear realisation of M-theory we compute all possible forms, that is objects with totally antisymmetrised indices, that occur in four dimensions and above as well as all the 1-forms and 2-forms in three dimensions. In any dimension $D$, the $D-1$-forms lead to maximal supergravity theories with cosmological constants and they are in precise agreement with the patterns of gauging found in any dimension using supersymmetry. The $D$-forms correspond to the presence of space-filling branes which are crucial for the consistency of orientifold models and have not been derived from an alternative approach, with the exception of the 10-dimensional case. It follows that the gaugings of supergravities and the spacetime-filling branes possess an eleven dimensional origin within the $E_{11}$ formulation of M-theory. This and previous results very strongly suggest that all the fields in the adjoint representation of $E_{11}$ have a physical interpretation.
\end{abstract}




\section{Introduction}

The IIA [1] and IIB [2, 3, 4] supergravity theories are the low energy effective actions for the corresponding IIA and IIB string theories, while the eleven dimensional supergravity theory [5] is thought to be the low energy effective action for an as yet undefined theory called M-theory. By dimensionally reducing any of these three supergravity theories on tori one finds maximal supergravity theories in lower dimensions. One of the most surprising discoveries concerning supergravity theories was the realisation that the maximal one in four dimensions possesses a hidden $E_{7}$ symmetry [6]. Furthermore, the maximal supergravity theory in three dimensions has an $E_{8}$ symmetry and more generally the maximal theory in $D$ dimensions possesses an $E_{11-D}$ symmetry for $D \geq 3$ [7]. In addition, the IIB theory possesses an $S L(2, \mathbb{R})$ symmetry [2]. The scalar fields which are created by the dimensional reduction process belong to a coset, or non-linear realisation, based on the corresponding $E_{11-D}$ algebra with the local sub-algebra being the Cartan involution invariant sub-algebra. All these supergravity theories possess charged states which are rotated by these symmetries and their charges obey quantisation conditions [8]. This has lead to the conjecture $[9,10,11]$ that discrete versions of the above groups, denoted by $G(\mathbb{Z})$, are symmetries in string theory, e.g. the $S L(2, \mathbb{R})$ symmetry of the IIB supergravity [2] becomes an $S L(2, \mathbb{Z})$ discrete symmetry [11]. The IIA and IIB supergravity theories and their dimensional reductions are uniquely determined by virtue of the supersymmetry that they possess and as a result they contain all the perturbative and non-perturbative effects of the corresponding IIA and IIB string theories. It is for this reason that these supergravity theories have played such an important role in our understanding of string theory as their properties transcend any particular formulation of it.

All the supergravity theories mentioned above are maximal in that they are invariant under the largest possible number of supersymmetries, namely 32. They also possess no other dimensional parameters other than the Planck scale. In fact, even this parameter can be absorbed into the fields such that it is absent from the equations of motion. We will refer to such theories as massless maximal supergravity theories. Indeed, their uniqueness rests on the absence of other dimensionful parameters. There are however, other theories that are also invariant under 32 supersymmetries, but possess additional dimensionful parameters; in this paper we will refer to these theories as massive maximal supergravity theories. These can be viewed as deformations of the massless maximal theories. However, unlike 
the massless maximal supergravity theories they can not in general be obtained by a process of dimensional reduction and in each dimension they have been determined by analysing the deformations that the corresponding massless maximal supergravity admits. The first example of such a theory was found in four dimensions [12], and it results from gauging an $S O(8)$ subgroup of the global symmetry $E_{7}$. The highest dimension for which a massive deformation is allowed is ten, and the corresponding massive theory was discovered by Romans [13]. This theory possesses a single additional mass parameter and can be thought of as a deformation of the IIA supergravity theory in which the two-form receives a mass via a Higgs mechanism. The number of maximal massive theories one finds increases rapidly as one considers lower and lower dimensions. These theories generically possess a local gauge symmetry carried by vector fields that can be thought of as part of the symmetry group $G$ of the corresponding maximal supergravity theory and have potentials for the scalars fields which contain the dimensionful parameters as well as a cosmological constant. Another typical feature of massive maximal supergravities is that their field content is not usually the same as their massless counterparts. As an example consider the five-dimensional $S O(6)$ gauged supergravity [14]. While the massless maximal supergravity theory [7, 15] contains 27 abelian vectors, the gauged one describes 15 vectors in the adjoint of $S O(6)$, as well as 12 massive tensors satisfying self-duality conditions. One can regard this as an example of the rearrangement of degrees of freedom induced by the Higgs mechanism.

In recent years there have been a number of systematic searches for massive maximal supergravity theories and in particular in dimensions from nine to three all such theories that possess a local gauge group were given in references $[16,17,18,19,20,21,22]$. We will refer to theories that possess a local gauge group as gauged maximal supergravity theories. It is believed that all massive maximal supergravity theories are gauged supergravities with the possible exception of Romans theory, which is not so much an exception but more a singular case. Although some of the massive maximal theories can be seen as arising from Scherk-Schwarz dimensional reductions, or from compactifications with fluxes turned on, there are many cases in which a higher dimensional supergravity origin is lacking. This can be seen already in ten dimensions, where the Romans theory can not be derived by a dimensional reduction of the eleven-dimensional supergravity theory. Hence, unlike for the maximal massless supergravities there is in general no higher dimensional origin for the massive maximal theories in terms of compactification of the eleven and ten dimensional massless ones and as a result there has been no systematic understanding of massive 
maximal supergravity theories. One of the main points of this paper is to show that $E_{11}$ does provide a systematic understanding and an eleven-dimensional origin of all massless and massive maximal supergravity theories.

In [23] it has been conjectured that an extension of eleven dimensional supergravity can be described by a non-linear realisation based on the group $E_{11}$. This conjecture was inspired by the result [24] that eleven dimensional supergravity itself can be formulated as a non-linear realisation of an algebra and that $E_{11}$ is the smallest Kac-Moody algebra which contains this algebra. This non-linear realisation in 11 dimensions naturally gives rise to both a 3 -form and a 6-form, and the resulting field equations are first order duality relations, whose divergence reproduces the 3-form field equations of 11-dimensional supergravity. Similarly, the graviton appears together with a dual graviton [23]. Seen from the perspective of the $E_{11}$ non-linear realisation, dimensional reduction on tori reveals bigger and bigger symmetries, but such symmetries are already present in the uncompactified theory. Compactifying more dimensions corresponds from this view point to choosing a vacuum in which a bigger subgroup of $E_{11}$ becomes manifest.

In [25] it was shown that $E_{11}$ also describes the IIA and IIB theories, where again all the fields appear together with their magnetic duals and therefore satisfy duality relations. The $E_{11}$ non-linear realisation appropriate to a $D$ dimensional theory requires a choice of $A_{D-1}$ sub-algebra which can be found by deleting nodes in the $E_{11}$ Dynkin diagram. It turns out that this sub-algebra is associated in the non-linear realisation with the $D$-dimensional gravity sector of the theory and as such the line of nodes corresponding to the $A_{D-1}$ subDynkin diagram is called the gravity line. This a reminiscent of a choice of vacuum and it distinguishes the gravity fields from the other fields in the non-linear realisation which are classified according to the $A_{D-1}$ sub-algebra. In eleven dimensions there is only one such $A_{10}$ algebra, but in ten dimensions there are two choices corresponding to the IIA and IIB supergravity theories. In lower dimensions one again finds only one choice of $A_{D-1}$ sub-algebra. Indeed it is remarkable to see the fields of the adjoint representation of $E_{11}$ decomposed in terms of the $A_{10}$ subalgebra corresponding to the IIB theory and observe that the first entries are precisely those of the fields of IIB supergravity plus their duals $[26]$.

As for any Kac-Moody algebra, $E_{11}$ contains an infinite number of generators for which no mathematical classification has been known, and consequently the field content of the $E_{11}$ non-linear realisation contains infinitely many fields in addition to those of the usual 
formulations of massless maximal supergravity theories. Even for some years after the conjecture of reference [23] the physical meaning of any of the fields in the adjoint representation of $E_{11}$ beyond those normally associated to the massless maximal supergravity theories was unclear. An exception was the field $A_{a_{1} \ldots a_{10}, b, c}$ of mixed symmetry, which occurs in the eleven-dimensional non-linearly realised theory just beyond the fields of eleven dimensional supergravity; this field upon dimensional reduction to ten dimensions gives rise to a nine-form. The massive IIA theory of Romans can be accounted for by introducing a nine-form [27, 28], whose field-strength is dual to Romans' cosmological constant, and in $[29,26]$ it was shown that the $E_{11}$ non-linear realisation in a 10-dimensional IIA background with non-vanishing 10-form field strength reproduces Romans' theory. Therefore $E_{11}$ provides an 11-dimensional origin for the massive IIA theory.

In order to see the $E_{11-D}$ symmetry as arising from dimensional reduction of the traditional formulations of the massless maximal supergravity theories it is crucial that the forms of rank higher than $[D / 2]-1$ be replaced by their electromagnetic duals. As an example, the dimensional reduction of the 3-form of 11-dimensional supergravity down to 4 dimensions gives rise to seven 2-forms, and these have to be dualised to scalars in order to see the whole $E_{7}$ symmetry arising. As we have mentioned the $E_{11}$ non-linear realisation automatically contains all the propagating fields and their magnetic duals and so when carrying out the dimensional reduction of these formulations it is unnecessary to introduce any additional fields, which is consistent with the fact that the symmetries that are found upon dimensional reductions are encoded in the uncompactified theory. Such democratic formulations have been useful in a number of different contexts; just before reference [23], in [30] it was shown that the IIA supersymmetry algebra admits a democratic formulation, in which all the RR-fields are introduced together with their magnetic duals. In this formulation, the field-strength of the RR 9-form can have any constant value, and therefore the algebra describes both the massless and the massive IIA theories. Also in order to classify $[18,19,20,21,22]$ the massive maximal supergravities it is necessary to dualise some of the fields of the corresponding massless theory. Indeed, a program of adding a hierarchy of higher tensor gauge fields in the context of gauging was begun in [31]. The systematic dualisation of the traditional fields of supergravity was first advocated in [32], were a non-linear realisation of the gauge sector of the maximal supergravity theories was given. The use of forms to encode the indices of the gauge fields resulted in a graded algebra which has so far not been generalised to include gravity. 
The $E_{11}$ formulation of the IIB theory was also found to contain three 8-forms in the triplet of $S L(2, \mathbb{R})$ as well as six space-filling 10-forms in the quadruplet and the doublet. In $[33,34]$ it was shown that the supersymmetry algebra allows the inclusion of a triplet of 8-forms dual to the scalars, while in an unexpected development [35] it was found that, provided one introduced the duals of all the propagating fields, the supersymmetry algebra of the IIB supergravity theory also allows a quadruplet and a doublet of 10-form fields. Indeed these forms are precisely the fields predicted by the $E_{11}$ formulation of the IIB theory, and furthermore it was later shown [36] that the IIB gauge algebra derived from $E_{11}$ precisely agrees with the one resulting from supersymmetry in [35]. The motivation of reference [35], which followed the results of [37], was to determine the multiplet to which the RR 10-forms associated to D9-branes belong. The D9-branes are spacetime-filling, and thus can not be consistently introduced in the IIB string theory because they have non-vanishing RR charge. This charge multiplies a 10-form gauge field in the Wess-Zumino term and its variation can not be canceled as 10-forms have no field strength. Nevertheless, they play a crucial role in constructing type-I string theory as the orientifold projection of IIB, which has the role of canceling the overall RR charge. A similar analysis was applied to the IIA supergravity theory [38] and it was found that the supersymmetry algebra closes if two 10-forms are added, a result that is in complete agreement with the predictions of the $E_{11}$ formulation of the IIA theory.

Thus the meaning of a few more fields of the $E_{11}$ non-linear realisation beyond the supergravity approximation was found. However, more recently a partial classification of all the fields contained in the $E_{11}$ non-linear realisation associated with the adjoint representation has been found [39]. As with any Kac-Moody algebra, the generators of $E_{11}$ arise from multiple commutators of the so called Chevalley generators. These are contained in the generators $K^{a}{ }_{b}$ of the $A_{10}$ sub-algebra when taken together with the generators $R^{a b c}$, associated with the three form field in eleven dimensional supergravity. Consequently, all the generators in $E_{11}$ arise from multiple commutators of $K^{a}{ }_{b}$ and $R^{a b c}$. The level of a generator is just the number of times the generator $R^{a b c}$ occurs in the commutator required to create that generator. It follows that a generator of level $l$ has $3 l$ indices and that the generators of a given level belong to representations of $A_{10}$. Of course it can, and does occur, that some generators can carry blocks of eleven anti-symmetrised indices. We note that these blocks do not transform under $A_{10}$. In reference [39] all generators without any blocks of ten and eleven anti-symmetrised indices were found. It turned out that in this 
sector there was essentially a void and the very few generators that did occur corresponded to all the infinitely many possible dual descriptions of the physical degrees of freedom of the eleven dimensional supergravity theory, namely the 3-form and the graviton.

By definition, the remaining fields contain at least one set of ten or eleven antisymmetric indices. Although the blocks of eleven indices do not transform under the $A_{10}$ sub-algebra, they do nonetheless have a significance, an example being the 10-forms in ten dimensional IIB theory. When the generators are viewed in this way they all carry indices and therefore the corresponding fields are all subject to local gauge transformations. This is consistent with the belief that all the $E_{11}$ transformations become local when combined with the conformal group. From this point of view, the infinite number of fields that are not dual to the 3-form and the graviton all give rise to no propagating on-shell degrees of freedom, but nonetheless have a physical meaning.

In this paper we want to extend the analysis of [39]. In particular, we determine all the forms, that is fields with completely antisymmetric indices, that occur in the $E_{11}$ non-linear realisation in dimensions three and above. We do this by considering the eleven dimensional theory and finding all fields that can give rise to forms in the lower dimensional theory using dimensional reduction. This is the same result that we would obtain by considering the forms arising from the $E_{11}$ non-linear realisation directly in the dimension of interest. However, the former approach has the advantage of providing an eleven-dimensional origin of all the forms. Remarkably, there is only a finite number of fields that can generate forms in any dimension above two. We compute all possible forms that occur in four dimensions and above as well as all the 1-forms and 2-forms in three dimensions. In any dimension $D$, all the forms of rank less than $D-1$ lead to the fields of the corresponding maximal supergravity in a democratic formulation where all the fields appear together with their magnetic duals. The $D-1$-forms have field-strengths which are dual to mass parameters, whose non-vanishing values lead to massive maximal supergravity theories. The classification of these $D-1$ forms arising from $E_{11}$ is in precise agreement with the the massive maximal supergravities $[16,19]$ found in any dimension using supersymmetry. It follows that one obtains a classification of all maximal supergravity theories and that they possess an eleven dimensional origin within the $E_{11}$ formulation of M-theory. However, this eleven dimensional origin can and often does arise from fields in the non-linear realisation that are beyond the supergravity approximation and in this case the corresponding massive theory can not be obtained as a dimensional reduction from the usual eleven dimensional 
supergravity theory. Our analysis reveals in which cases the massive theory admits a higher dimensional supergravity origin, and one can thus show why some theories have a IIB supergravity origin but not an eleven-dimensional supergravity origin and vice-versa. We also find, except in three dimensions, all possible $D$-forms which correspond to the presence of space-filling branes. These are crucial for the consistency of orientifold models and have not been derived from an alternative approach, with the exception of the tendimensional case. In the classification of the $D-1$ and $D$ forms for the lower dimensions one requires fields that correspond to roots of $E_{11}$ which have rather negative length squared and in some cases multiplicities more than one. Hence, one is using in these calculations properties of the $E_{11}$ Kac-Moody algebra that are very far from those one finds in affine or finite Lie algebras. The $E_{11}$ non-linear realisation in $D$ dimensions contains the fields corresponding to the degrees of freedom of the maximal supergravity theory, as well as fields for all the infinitely many possible dual descriptions of these propagating degrees of freedom, together with an infinite number of fields having at least one set of $D-1$ or $D$ indices completely antisymmetrised. Among the latter, there is always a finite number of $D-1$ and $D$-forms.

The plan of the paper is as follows. In Section 2 we classify all the 11-dimensional fields in $E_{11}$ that can give rise to forms in any dimension above three, as well as one-forms and two-forms in three dimensions. In Section 3 we summarise the results of the various dimensional reductions. Section 4 is devoted to a detailed analysis of each dimension separately. In Section 5 we show how the dynamics and in particular the gauging results from the non-linear realisation, focusing on the five-dimensional case. Section 6 contains the conclusions.

\section{The structure of the eleven dimensional algebra}

In order to proceed with the analysis of the eleven dimensional field content resulting from the non-linear realisation of $E_{11}$, let us first review some of the basic ideas underlying the $E_{11}$ construction of the low-energy effective action of M-theory that will be relevant for the remaining of the paper. In [23] it was conjectured that an extension of eleven dimensional supergravity can be described by a non-linear realisation based on the group $E_{11}$. $E_{11}$ was also shown to give rise to non-linear realisations that are extensions of IIA [23] and IIB [25] supergravities, consistent with the conjecture that $E_{11}$ is a symmetry of the low-energy 
effective action of M-theory [23].

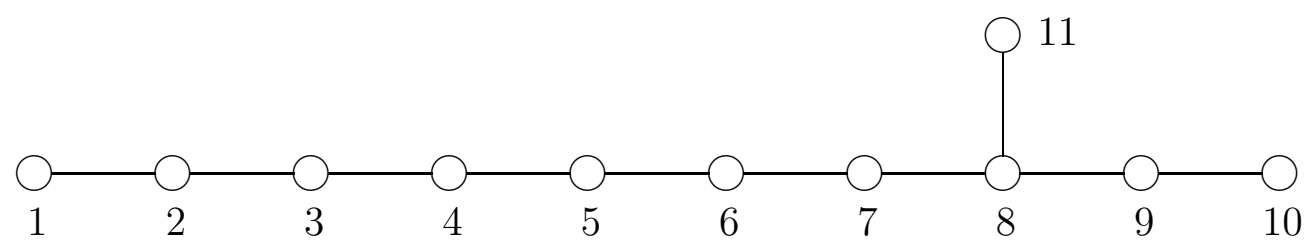

Figure 1: The $E_{11}$ Dynkin diagram corresponding to 11-dimensional supergravity.

$E_{11}$ is the Kac-Moody algebra resulting from the Dynkin diagram of Fig. 1. The delation of the node 11 in the diagram results in the $A_{10}$ or $S L(11, \mathbb{R})$ algebra that gives rise in the non-linear realisation to the eleven dimensional gravity sector of the theory. As previously discussed, the horizontal line in the diagram is called the gravity line. The simple roots of $E_{11}$ are the simple roots $\alpha_{i}, i=1, \ldots, 10$ of $A_{10}$ as well as the simple root $\alpha_{11}$ which is given by

$$
\alpha_{11}=x-\lambda_{8}
$$

Here $x$ is orthogonal to the roots $\alpha_{i}, i=1, \ldots, 10$ of $A_{10}$, and $\lambda_{i}, i=1, \ldots, 10$ are the fundamental weights of $A_{10}$. As $\alpha_{11} \cdot \alpha_{11}=2$ and $\lambda_{8} \cdot \lambda_{8}=\frac{24}{11}$, one gets

$$
x^{2}=-\frac{2}{11}
$$

Using equation (2.1), any root $\alpha$ of $E_{11}$ can be written as

$$
\alpha=\sum_{i=1}^{10} n_{i} \alpha_{i}+l \alpha_{11}=l x-\Lambda
$$

where

$$
\Lambda=l \lambda_{8}-\sum_{i=1}^{10} n_{i} \alpha_{i}
$$

is in the weight space of $A_{10}$. The integer $l$, denoting the number of times the simple root $\alpha_{11}$ appears in the simple root decomposition of $\alpha$, is called the level, and the strategy $[40,41,42]$ is to analyse the generators of $E_{11}$ level by level in terms of the representations of $A_{10}$.

We wish to decompose the adjoint representation of $E_{11}$ in terms of representations of $A_{10}$. A necessary condition for the occurrence of a representation of $A_{10}$ with highest 
weight $\sum_{j} p_{j} \lambda_{j}$, where $p_{j}$ are known as Dynkin indices, is that this weight arises in a root of $E_{11}$ in eq. (2.3). In other words, there exists a $\Lambda$ such that

$$
\Lambda=\sum_{j} p_{j} \lambda_{j}
$$

Taking the scalar product with $\lambda_{i}$ implies that

$$
\sum_{j} p_{j} A_{j i}^{-1}=l A_{8 i}^{-1}-n_{i}
$$

where we have used the equation

$$
\left(A_{j k}\right)^{-1}=\left(\lambda_{j}, \lambda_{k}\right)
$$

valid for any simply laced finite dimensional semi-simple Lie algebra. Here $\left(A_{i k}\right)^{-1}$ is the inverse Cartan matrix, which in the case of $A_{10}$ is given by

$$
\left(A_{j k}\right)^{-1}=\left\{\begin{array}{ll}
\frac{j(11-k)}{11}, & j \leq k \\
\frac{k(11-j)}{11}, & j \geq k
\end{array} .\right.
$$

This equation places strong restrictions on the possible $p_{j}$, and so the representations of $A_{10}$, that can occur at a given level. Using eqs. (2.3), (2.5) and (2.7) one obtains

$$
\alpha^{2}=-\frac{2}{11} l^{2}+\sum_{i, j} p_{i}\left(A_{i j}\right)^{-1} p_{j}
$$

The fact that $E_{11}$ is a Kac-Moody algebra with symmetric Cartan matrix imposes the constraint [43]

$$
\alpha^{2}=2,0,-2,-4 \ldots
$$

on the roots, and therefore each root obeys eq. (2.9) with the constraint (2.10). Observe that not any root which is a solution of these two equations necessarily leads to a generator of the $E_{11}$ algebra, because further constraints come from the Serre relations. Similarly, the multiplicity of a given root can be higher than 1, so that it corresponds to more generators associated to that root. The solutions with multiplicity zero, that is the ones ruled out by the Serre relations, are very rare as can be seen from the tables of Refs. [44, 26].

It is convenient to swap the Dynkin indices $p_{j}$ for the indices $q_{j}$ given by

$$
q_{j}=p_{11-j}
$$


in order to make more transparent the relation between fields and generators. We recall the generators of $E_{11}$ that occur for the first three levels [23]. The level zero generators correspond to the generators $K^{a}{ }_{b}$ of $A_{10}$. At level one we have

$$
R^{a b c}, \quad l=1, q_{3}=1
$$

at level two

$$
R^{a_{1} \ldots a_{6}}, \quad l=2, q_{6}=1
$$

and at level three

$$
R^{a_{1} \ldots a_{8}, b}, \quad l=3, q_{1}=1, q_{8}=1 .
$$

At level three we also find $R^{a_{1} \ldots a_{9}}, q_{9}=1$, but this generator has multiplicity zero and so does not actually occur in the $E_{11}$ algebra. $E_{11}$ is defined as the multiple commutators of its Chevalley generators, subject to the Serre relations. The multiple commutators of the level zero Chevalley generators lead to the generators $K^{a}{ }_{b}$ of $S L(11, \mathbb{R})$, while the multiple commutators of these with the level one Chevalley generator lead to $R^{a b c}$ of eq. (2.12). All the other positive level generators are then found from multiple commutators of $R^{a b c}$ subject to the Serre relation, and the level is the number of times the generator $R^{a b c}$ occurs in the commutators. This implies that the generators at level $l$ have $3 l$ upper indices. The same applies to the construction of all the negative root generators, which have $3 l$ lower indices when written as representations of $A_{10}$, corresponding to negative level.

The non-linear realisation is constructed from a group element of $E_{11}$ which is subject to a local transformation that can be used to put the group element in the Borel subgroup. The Borel subgroup is the one generated by the Cartan subalgebra and the generators associated with the positive roots. As a result, there is a one-to-one correspondence between the fields of the theory and the generators of $E_{11}$ with non-negative level. At level zero, this results in the description of gravity as a non-linear realisation [45, 46], and the level zero field is therefore the graviton. The generator at level 1 in (2.12) corresponds to the 3-form of 11-dimensional supergravity, the one at level 2 in (2.13) to its 6-form dual, and the one at level 3 given in (2.14) corresponds to the dual graviton [23]. A generator with $q_{j}=1$ has $j$ antisymmetrised indices, and for each non-vanishing Dynkin index $q_{j}$, the generator possesses $q_{j}$ blocks of $j$ antisymmetric indices. Therefore a given set of $q_{j}$ 's corresponds to the $A_{10}$ irreducible representation determined by a Young Tableaux with a number of columns equal to $\sum_{j} q_{j}$, the column corresponding to each given $j$ having $j$ boxes. For 
readers more used to the Dynkin indices $p_{j}$, the relation of these with the $q_{j}$ 's is given in eq. (2.11). In the non-linear realisation, such a generator gives rise to a corresponding gauge field with the same index structure. The sum of all the indices of the fields is thus equal to 3l. This way of associating gauge fields to generators takes account of the possibility that some fields may have blocks of 11 antisymmetric indices. These can be determined by the relation

$$
11 n+\sum_{j} j q_{j}=3 l
$$

where $n$ is the number of columns with 11 boxes [39]. In the rest of the paper we will denote a field with $j$ antisymmetric indices by a suffix $j$, and the 11-dimensional fields will be also hatted to distinguish them from the lower dimensional ones. The fields corresponding to the generators in eqs. (2.12), (2.13) and (2.14) are therefore denoted as

$$
\hat{A}_{3} \quad, \quad \hat{A}_{6} \quad, \quad \hat{A}_{8,1} .
$$

We now review the main result of [39], where all the generators of $E_{11}$ with no 10 or 11 antisymmetric indices were classified. Substituting eq. (2.15) with $n=q_{10}=0$ in (2.9) one gets

$$
\alpha^{2}=\frac{1}{9}\left[\sum_{i} i(9-i) q_{i}^{2}+2 \sum_{i<j} i(9-j) q_{i} q_{j}\right] .
$$

The solutions of this equation corresponding to roots are

$$
\begin{array}{ll}
q_{3}=1 & q_{9}=m, \\
q_{6}=1 & q_{9}=m
\end{array}
$$

and

$$
q_{1}=1 \quad q_{8}=1 \quad q_{9}=m
$$

for any non-negative integer $m$. The solution $q_{9}=m$ with all the other Dynkin indices zero turns out not to be a root because of the Serre relations. The solutions of the form (2.18) correspond to the infinite dual descriptions of a 3-form in eleven dimension, while the solutions of the form (2.19) correspond to the infinite dual descriptions of the graviton. Therefore, the outcome of this analysis is that the adjoint representation of $E_{11}$ contains generators corresponding to the infinite possible dual descriptions of the 3 -form and the graviton of eleven-dimensional supergravity, which are the bosonic degrees of freedom of this theory [5]. 
In this paper we want to consider the dimensional reduction of the 11-dimensional fields in the adjoint representation of $E_{11}$. In particular, we are only interested in the 11-dimensional fields that give rise to forms, i.e. fields with only one set of completely antisymmetric indices. In $D$ dimensions, we divide the forms in three different groups:

- all the ones of rank less than $D-1$, which we call propagating forms because their field equations propagate degrees of freedom;

- the ones of rank $D-1$, whose $D$-form field strengths are dual to cosmological constants, and are therefore associated to the massive deformations of the corresponding $D$-dimensional supergravity;

- the ones of rank $D$, that have no dynamics and no field equations, but are typically associated to spacetime-filling branes.

The derivation makes use of the fact that a field of the form $\hat{A}_{p, q}$ can give rise to a form after compactification only if all the $p$ 's or all the $q$ 's are internal indices. This generalises to the case in which there are more than two sets of antisymmetric indices. For example, a form in three dimensions can only arise from fields that have one set of 9, 10 or 11 antisymmetric indices or no sets of indices of this kind, because a maximum of 8 antisymmetric indices can be internal.

The forms of the first type can only arise from the fields that were considered in [39], corresponding to the solutions (2.18) and (2.19). In particular, the only fields that give rise to forms for any dimension higher than 2 are listed in Table 1. All the other fields considered in [39] have at least two sets of 9 antisymmetric indices, and so they can generate forms only in two dimensions.

The $D-1$ forms in $D$ dimensions can arise from the fields of Table 1, as well as from field that have one set of 10 antisymmetric indices in 11 dimensions. Therefore, in order to determine all the possible $D-1$-forms that arise in any dimension higher than 2 , we have to classify all the generators of $E_{11}$ that have $n=q_{9}=0$ and $q_{10}=1$ in eq. (2.15). Indeed, there can not be any set of 11 antisymmetric indices, and one can only allow for one set of 10 antisymmetric indices. Since all the other sets of indices have to be internal after dimensional reduction, and we are interested in compactifications to three dimensions and above, we exclude the possibility that there is also any set of 9 antisymmetric indices. 


\begin{tabular}{|c||c|}
\hline $\mathrm{D}$ & field \\
\hline 10 & $\hat{g}^{1}$ \\
& $\hat{A}_{3}$ \\
& $\hat{A}_{6}$ \\
& $\hat{A}_{8,1}$ \\
\hline 8 & $\hat{A}_{9,3}$ \\
\hline 5 & $\hat{A}_{9,6}$ \\
\hline 3 & $\hat{A}_{9,8,1}$ \\
\hline
\end{tabular}

Table 1: Table listing all the 11-dimensional fields that give rise to propagating forms (i.e.forms of rank less than $D-1$ ) after dimensional reduction. The first column indicates the highest dimension where these fields give rise to forms.

Substituting $n=q_{9}=0, q_{10}=1$ in eq. (2.9) one gets

$$
\alpha^{2}=\frac{1}{9}\left[-10+\sum_{i} i(9-i) q_{i}^{2}-2 \sum_{i} i q_{i}+2 \sum_{i<j} i(9-j) q_{i} q_{j}\right] \text {. }
$$

We determine all the possible solutions of eq. (2.10) using this expression for $\alpha^{2}$. Since this expression is bounded from below, there are only a finite number of solutions, and after substituting in (2.20) a few values for $q_{i}$ 's, the reader can see how one can proceed by inspection. Not all these solutions actually correspond to roots, but it turns out that the multiplicity of all the solutions that we find has been derived in the literature [44]. There are no roots of this type with multiplicity higher than 1, and there are only two solutions corresponding to multiplicity zero, namely $q_{2}=q_{10}=1$ (level 4 ) and $q_{5}=q_{10}=1$ (level 5). The compete set of fields that we find is listed in Table 2.

Finally, in order to determine all the possible $D$-forms arising after dimensional reduction, in addition to the ones arising from the fields in Tables 1 and 2, one has to classify all the generators with $n=1$ and $q_{10}=q_{9}=0$ in eq. (2.15). Indeed, only one set of 11 indices is allowed, and once this is done, we have to restrict the fields to not having 10 or 9 indices if we want to generate forms after compactification. Substituting $n=1, q_{10}=q_{9}=0$ in eq. (2.9) one gets

$$
\alpha^{2}=\frac{1}{9}\left[-22+\sum_{i} i(9-i) q_{i}^{2}-4 \sum_{i} i q_{i}+2 \sum_{i<j} i(9-j) q_{i} q_{j}\right] .
$$

This expression is bounded from below, and therefore there are a finite number of solutions 


\begin{tabular}{|c||c|}
\hline $\mathrm{D}$ & field \\
\hline 10 & $\hat{A}_{10,1,1}$ \\
\hline 7 & $\hat{A}_{10,4,1}$ \\
\hline 5 & $\hat{A}_{10,6,2}$ \\
\hline 4 & $\hat{A}_{10,7,1}$ \\
& $\hat{A}_{10,7,4}$ \\
& $\hat{A}_{10,7,7}$ \\
\hline 3 & $\hat{A}_{10,8}$ \\
& $\hat{A}_{10,8,2,1}$ \\
& $\hat{A}_{10,8,3}$ \\
& $\hat{A}_{10,8,5,1}$ \\
& $\hat{A}_{10,8,6}$ \\
& $\hat{A}_{10,8,7,2}$ \\
& $\hat{A}_{10,8,8,1}$ \\
& $\hat{A}_{10,8,8,4}$ \\
& $\hat{A}_{10,8,8,7}$ \\
\hline
\end{tabular}

Table 2: Table listing all the fields containing one set on 10 antisymmetric indices giving rise to forms in $D$ dimensions, the first column indicating the highest dimension where this occurs.

of eq. (2.10). We determine all the possible solutions of eq. (2.10) using this expression for $\alpha^{2}$, but it turns out that not for all the solutions the corresponding multiplicity is known. All the solutions of which we do not know the multiplicity have $q_{8} \geq 1$, and thus the corresponding fields would give rise to $D$-forms only for $D=3$. Consequently, we are able to classify all the possible solutions, with the corresponding multiplicity, for any dimension greater than 3. The result of this analysis is shown in Table 3, whose last column denotes the multiplicity of the field. We leave the classification of the 3 -forms in 3 dimensions as an open project.

As is evident from the above, the higher level fields contained in the non-linear realisation of $E_{11}$ generically have mixed symmetry. One may expect that each block of indices has associated with it a local gauge transformation and this should also apply to blocks of 11 indices. Writing the field with $3 l$ indices incorporates the correct gauge transformations. We note that raising and lowering with the $\epsilon$ symbol and then interpreting the gauge transformations would lead to different results. In this respect, a field with a block of 11 indices carries the corresponding gauge transformations as so does not possess an invariant 


\begin{tabular}{|c||c|c|}
\hline $\mathrm{D}$ & field & $\mu$ \\
\hline 10 & $\hat{A}_{11,1}$ & 1 \\
\hline 8 & $\hat{A}_{11,3,1}$ & 1 \\
\hline 7 & $\hat{A}_{11,4}$ & 1 \\
& $\hat{A}_{11,4,3}$ & 1 \\
\hline 6 & $\hat{A}_{11,5,1,1}$ & 1 \\
\hline 5 & $\hat{A}_{11,6,1}$ & 2 \\
& $\hat{A}_{11,6,3,1}$ & 1 \\
& $\hat{A}_{11,6,4}$ & 1 \\
& $\hat{A}_{11,6,6,1}$ & 1 \\
\hline \multirow{2}{*}{4} & $\hat{A}_{11,7}$ & 1 \\
& $\hat{A}_{11,7,2,1}$ & 1 \\
& $\hat{A}_{11,7,3}$ & 2 \\
& $\hat{A}_{11,7,4,2}$ & 1 \\
& $\hat{A}_{11,7,5,1}$ & 1 \\
& $\hat{A}_{11,7,6}$ & 2 \\
& $\hat{A}_{11,7,6,3}$ & 1 \\
& $\hat{A}_{11,7,7,2}$ & 1 \\
& $\hat{A}_{11,7,7,5}$ & 1 \\
\hline
\end{tabular}

Table 3: Table listing all the fields containing one set on 11 antisymmetric indices giving rise to forms in $D$ dimensions. The first column indicates the highest dimension where this occurs, while the last column indicates the multiplicity of the field.

field strength, and as such can not lead to a propagating degree of freedom.

\section{Listing of forms in lower dimensions}

Before considering each dimension separately, in this section we summarise the results, which are collected in Table 5 at the end of this paper. From the dimensional reduction of the fields in Tables 1,2 and 3, we determine all the forms that arise in any dimension higher than three, as well as the 1-forms and 2-forms in three dimensions. As already noticed, the same results can be found by using the $E_{11}$ non-linear realisation corresponding to the dimension of interest, that is taking the appropriate gravity line. In each dimension, $E_{11}$ decomposes in $S L(D, \mathbb{R}) \otimes G$, where the first group corresponds to the non-linear realisation of gravity, and the second is the internal symmetry of the given supergravity theory. In 
all the $E_{11}$ Dynkin diagrams drawn in this paper, Figs. 1-10, $S L(D, \mathbb{R})$ corresponds to the horizontal line, while $G$ corresponds to the Dynkin diagram contained in the box. Denoting by $\mathbf{r}_{\mathbf{n}}$ the representation of $G$ to which the $n$-forms belong in a given dimension $D$ less than 10, one can see from Table 5, using the decomposition rules of [47], that

$$
\mathbf{r}_{1} \otimes \mathbf{r}_{\mathbf{n}} \supset \mathbf{r}_{\mathbf{n}+\mathbf{1}}
$$

This is a completely general result from $E_{11}$, and it says that it any dimension lower than 10 the 1 -forms are the basic building blocks of the algebra. More technically, the Chevalley generators are contained in the gravity sector, the internal symmetry algebra and the 1forms, and as such these must generate the full $E_{11}$ algebra. This is transparent if one looks at the corresponding Dynkin diagrams.

The propagating forms (i.e. the forms of rank less than $D-1$ ) all originate from Table 1. For all the forms of rank less that $D-2$ one has

$$
\mathbf{r}_{\mathbf{n}}=\left(\mathbf{r}_{\mathbf{D}-\mathbf{2}-\mathbf{n}}\right)^{*}
$$

This allows the duality relation of the corresponding fields. Indeed, the field strengths of the $n$-forms and the $D-2-n$-forms in $D$ dimensions are related by

$$
F_{n+1}=* F_{D-n-1}
$$

and since the $*$ operator also contains the charge conjugation in the internal sector, this leads to eq. (3.2). In even dimension, the field-strengths of the forms of rank $\frac{D}{2}-1$ satisfy self-duality relations. Finally, The forms of rank $D-2$, which are dual to scalars, always belong to the adjoint of $G$. Since the scalars parametrise the manifold $G / H$, with $H$ the maximal compact subgroup of $G$, so that only $\operatorname{dim} G-\operatorname{dim} H$ scalars propagate, there are $\operatorname{dim} H$ field-strengths of these $D-2$-forms that vanish identically. This result was derived from supersymmetry in $[33,34]$ in the 10 -dimensional IIB case.

Observe that in any dimension above three, the number of scalars is determined by the formula

$$
\operatorname{scalars}_{D}=\operatorname{scalars}_{D+1}+1+\operatorname{dim} \mathbf{r}_{\mathbf{1}}^{\mathbf{D}+\mathbf{1}}
$$

where $\mathbf{r}_{\mathbf{1}}^{\mathbf{D + 1}}$ is the representation of the 1 -forms in $D+1$ dimensions. This is due to the fact that the scalars arise from the scalars, the metric and the 1-forms in one dimension higher. In three dimensions this formula has to be replaced by

$$
\text { scalars }_{3}=\text { scalars }_{4}+2+\operatorname{dim} \mathbf{r}_{1}^{4}
$$


where one extra scalar comes from the compactification of the four-dimensional dual graviton $A_{1,1}$. This is in agreement with the well-known fact that in three dimensions the vector arising from the four-dimensional graviton gives rise to an extra scalar after duality, but the difference is that $E_{11}$ encodes automatically all the duality relations. The same result can of course be seen as arising from 11 dimensions, where the 3 -form $\hat{A}_{3}$ gives rise to scalars in $D=8$ and below, the 6 -form $\hat{A}_{6}$ in $D=5$ and below, and the dual graviton $\hat{A}_{8,1}$ only in $D=3$. To summarise, once all the duality relations are taken into account, the results we find reproduce the well-known field content of all the maximal supergravity theories.

The forms of rank $D-1$ originate from Tables 1 and 2 . These fields have a $D$-form fieldstrength, and are therefore dual to constants, that are interpreted as mass deformations. We determine the representation of these forms in any dimension. This corresponds to the representation of the most general mass deformation of a gauged maximal supergravity, and indeed we show that our results precisely agree with the classification of all the possible deformations of maximal supergravities in any dimension [19]. In [27, 28] it was shown that the massive IIA theory [13] can be accounted for introducing a 9-form, whose field-strength is dual to Romans' cosmological constant. The resulting supersymmetry algebra [30] gives the supersymmetry algebra of massless IIA [1] in the limit in which the cosmological constant vanishes. In $[29,26]$ it was shown how the $E_{11}$ algebra corresponding to IIA automatically encodes a 9-form and thus the cosmological constant of Romans. In fact the Romans massive IIA theory has an 11-dimensional origin and the 9-form results from the dimensional reduction of the field $\hat{A}_{10,1,1}$ in Table 2 [48]. Our results show that this way of determining the masses is completely general, and, most remarkably, that any massive maximal supergravity theory has an 11-dimensional origin. If the $D-1$-form arises from one of the fields in Table 1, that is the 3-form, the metric and their duals, then the resulting massive supergravity can be derived as a compactification of the traditional 11-dimensional supergravity theory. However, if the originating field is not in Table 1, then the corresponding massive supergravity does not arise from a compactification of the traditional 11-dimensional supergravity, but it is a compactification of the 11-dimensional $E 11$ non-linear realisation. Similar statements apply to the $E_{11}$ formulation of the IIB theory, and one can find that the originating field sometimes belongs to the traditional fields of the IIB theory plus their duals, but not of the traditional 11-dimensional theory. More generally, the gauged supergravities that are known to have a higher dimensional 
origin in terms of the traditional formulations of supergravity theories correspond to mass parameters generated by the supergravity fields or their duals in the dimension of origin. As an example, the mass parameter corresponding to the $S O(6)$ gauged supergravity in five dimensions [14] can be shown to have a IIB supergravity origin, while it corresponds to the field $\hat{A}_{10,1,1}$ from the 11-dimensional perspective. This agrees with the fact that the five-dimensional theory arises from an $S^{5}$ compactification of IIB [49].

We finally consider the $D$-forms that arise in $D$ dimensions. These come from the dimensional reduction of all the fields in Tables 1,2 and 3. The 10-dimensional IIA and IIB cases were determined in [26] from $E_{11}$, and in [38, 35] imposing the closure of the supersymmetry algebra. Not only their number agrees precisely, but also the corresponding gauge algebra [36]. We determine the representations of these forms for any dimension greater than 3. None of these results was known in the literature for dimension lower than 10. As one can see from Table 3, some of the fields involved in this computation have multiplicity 2. This turns out to be essential for the $D$-forms to collect in representations of the internal symmetry group $G$. Although these fields are not propagating, they are in general associated to spacetime-filling branes. Spacetime-filling D-branes are a basic ingredient for the consistency of orientifold models, and we believe that the classification or the representations to which these D-branes belong will turn out to be relevant for a deeper understanding of non-perturbative string theory and M-theory. In [50] it was shown that $\kappa$-symmetry of the effective action of D9-branes in IIB implies that the charge of such branes, belonging to the quadruplet, have to lie in a particular conjugacy class that identifies a non-linear doublet inside the quadruplet. This is also essential in order to introduce a world-volume vector in the effective action [51]. It would be interesting to perform the same analysis in lower dimensions.

\section{Maximal supergravities in various dimensions}

In this section we determine all the forms that arise from the non-linear realisation of $E_{11}$ in any dimension. We consider each dimension separately, starting from the case of $D=10$. For any dimension we determine the propagating fields, as well as all the possible mass deformations, that arise as $D$-1-forms, whose $D$-form field strengths being dual to masses. We collect all the forms as representations of the internal symmetry group $G$ of the maximal supergravity under consideration. The spectrum and all the mass deformations that we find 
are in perfect agreement with the literature. We also determine, for any dimension greater than 3 , all the $D$-forms. Although these objects are non-propagating, they are associated to spacetime-filling branes, that play a crucial role in the construction of orientifold models. We show how these forms are grouped in representations of the internal symmetry group. Apart from the ten-dimensional case, these results were previously unknown. For any dimension we show the corresponding $E_{11}$ Dynkin diagram, where the horizontal nodes are associated to gravity. In this way of drawing the diagram, it is transparent how the internal symmetry arises in a given dimension: it corresponds to the nodes that are not connected to the gravity ones.

How the fields that occur in the dimensional reduction of a maximal supergravity theories correspond to the Dynkin diagram of the corresponding $E_{n}$ symmetries was discussed in [52], where it was also briefly noted that if one did this for all the fields in all the dimensionally reduced theories then it was natural to consider a rank eleven algebra associated with the dimensionally reduced theory. However, at no point was it suggested that this algebra could correspond to a symmetry in the eleven or ten dimensional theories.

\section{$\mathrm{D}=10$}

Type-IIA supergravity arises as a dimensional reduction of 11-dimensional supergravity on $S^{1}$. The bosonic sector of the theory contains a scalar parametrising $\mathbb{R}^{+}$, the metric, a vector, a 2 -form and a 3 -form. According to $E_{11}$, these objects appear in the algebra together with an infinite chain of dual fields. So for instance the 1-form appears together with a 7 -form, as well as an infinite chain of $A_{8,8, \ldots, 8,1}$ and $A_{8,8, \ldots, 8,7}$ fields, all related by dualities. All these fields arise from dimensional reduction of the 11-dimensional field content deduced from $E_{11}$. The Dynkin diagram associated to this theory is shown in Fig. 2 .

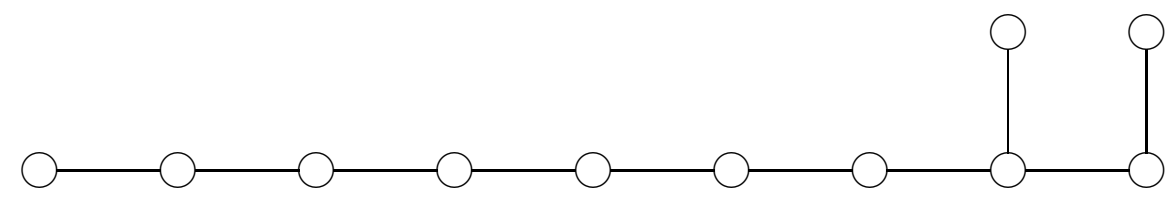

Figure 2: The $E_{11}$ Dynkin diagram corresponding to 10-dimensional IIA supergravity. 
Let us first consider all the forms associated to propagating fields. The result is

$$
\begin{gathered}
\hat{g}_{1}^{1} \rightarrow A_{1}, \quad \phi \\
\hat{A}_{3} \rightarrow A_{3}, A_{2} \\
\hat{A}_{6} \rightarrow A_{6}, A_{5} \\
\hat{A}_{8,1} \rightarrow A_{8}, A_{7},
\end{gathered}
$$

corresponding to the known propagating forms and their dual forms.

We now consider the 9 and 10-forms. From the first field in Table 2 one gets

$$
\hat{A}_{10,1,1} \rightarrow A_{9}
$$

while from the same field and from the first field in Table 3 one obtains two 10-forms,

$$
\hat{A}_{10,1,1} \rightarrow A_{10} \quad \hat{A}_{11,1} \rightarrow A_{10}^{\prime}
$$

The field-strength of the 9-form is dual to Romans' cosmological constant. The fact that $E_{11}$ can account for the mass deformation of Romans was shown in [29, 26], and the higher dimensional origin of the 9-form of eq. (4.2) given in [48]. In [38] it was shown that the supersymmetry algebra of IIA precisely reproduces the predictions of $E_{11}$ here summarised, and in particular that there are two 10-forms.

Before proceeding to analyse the lower dimensional cases, it is important to notice that the $E_{11}$ symmetry is compatible with a different 10-dimensional background, corresponding to type-IIB supergravity [25], describing in the bosonic sector the metric, two scalars parametrising the manifold $S L(2, \mathbb{R}) / S O(2)$, two 2-forms and a self-dual 4-form [2]. The Dynkin diagram associated to the IIB background is show in Fig. 3. It is manifest from the diagram that the theory possesses an $S L(2, \mathbb{R})$ internal symmetry, and that the 2forms belong to the $\mathbf{2}$ of such group. The propagating forms that arise are two scalars, a doublet of 2-forms and of dual 6-forms, as well as a self-dual 4-form, which is a singlet of $S L(2, \mathbb{R})$ and a triplet of 8 -forms dual to the scalars. The 8-forms are therefore in the adjoint of $S L(2, \mathbb{R})$, and the duality relation between the 8-forms and the scalars is such that a combination of the three 9-form field-strengths vanishes identically. As already anticipated, this is a completely general result: the $D-2$-forms dual to the scalars are in the adjoint of the internal symmetry group $G$, while the scalars parametrise the manifold $G / H$. Therefore the corresponding duality relation puts to zero $\operatorname{dim} H D-1$-form field 


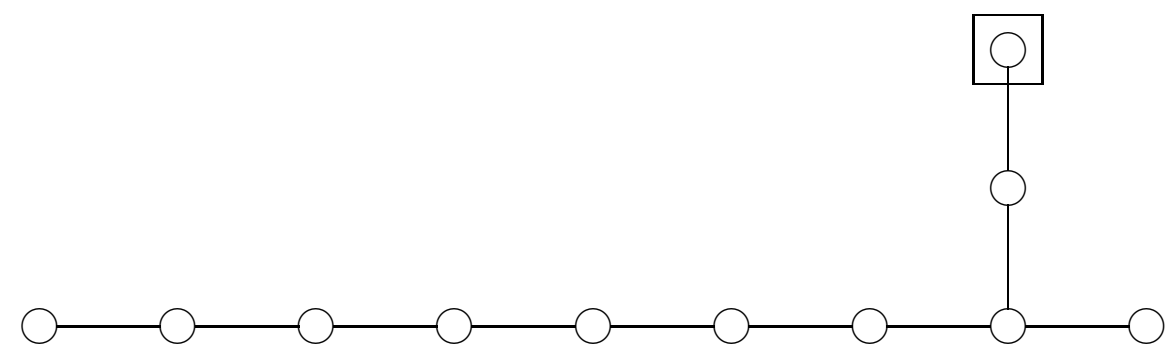

Figure 3: The $E_{11}$ Dynkin diagram corresponding to 10-dimensional IIB supergravity. The internal symmetry group is $S L(2, \mathbb{R})$.

strengths. $E_{11}$ also predicts the presence of a quadruplet and a doublet of 10 -forms. In [35] it was shown that these are precisely the forms contained in the IIB supersymmetry algebra. It was then shown that the gauge transformations derived in [35] imposing the closure of the supersymmetry algebra are exactly as predicted by $E_{11}$ [36].

Although the dimensional reductions of the IIA and IIB spectrum arising from $E_{11}$ are the same, we list in Table 4 the fields of the IIB spectrum that give rise to forms in nine and eight dimensions [26]. As we will see, this will resolve some ambiguities in assigning representations to the fields in eight dimensions. One can see from Table 4 that the fields with an even number of $\alpha$ indices have $4,8,12 \ldots$ spacetime indices, while the ones with an odd number of $\alpha$ indices have $2,6,10 \ldots$ spacetime indices. This result is completely general, and it is due to the fact that the generators are constructed from multiple commutators of $R^{a b, \alpha}$.

\section{$\mathrm{D}=\mathbf{9}$}

The three scalars of maximal massless 9-dimensional supergravity parametrise the manifold $\mathbb{R}^{+} \times S L(2, \mathbb{R}) / S O(2)$. The theory also contains the metric, a doublet and a singlet of vectors, a doublet of 2 -forms and a 3 -form. The $E_{11}$ Dynkin diagram corresponding to a 9-dimensional background is shown in Fig. 4. From the diagram one can indeed see that the non-abelian internal group is $S L(2, \mathbb{R})$, and there are a doublet and a singlet of vectors. This symmetry can either be seen as arising from 11 dimensions, with the last two nodes on the left of the diagram of Fig. 1 being associated with the coordinates of a two-torus, or as arising from IIB in 10 dimensions, with the last node of Fig. 3 corresponding to the coordinate of the circle. 


\begin{tabular}{|c||c|}
\hline D & IIB fields \\
\hline 9 & $g^{1}$ \\
& $A_{2}^{\alpha}$ \\
& $A_{4}$ \\
& $A_{6}^{\alpha}$ \\
& $A_{8}^{(\alpha \beta)}$ \\
& $A_{7,1}$ \\
& $A_{10}^{\alpha}$ \\
& $A_{10}^{(\alpha \beta \gamma)}$ \\
& $A_{9,1}^{\alpha}$ \\
\hline 8 & $A_{8,2}^{\alpha}$ \\
& $A_{9,2,1}^{\alpha}$ \\
& $A_{10,2}^{(\alpha \beta)}$ \\
& $A_{10,2}$ \\
& $A_{10,2}^{\prime}$ \\
& $A_{10,2,2}^{\alpha}$ \\
\hline
\end{tabular}

Table 4: Table listing all the fields of IIB that can give rise to forms in 9 and 8 dimensions, with the first column denoting the highest dimension for which this occurs. The upstairs indices are indices of $S L(2, \mathbb{R}), \alpha=1,2$.

We now list all the forms that arise from the 11-dimensional fields of Tables 1,2 and 3 as representations of $S L(2, \mathbb{R})$, denoting as usual by $\mathbf{r}_{\mathbf{n}}$ the representation to which the $n$-forms belong. The result is

$$
\begin{array}{lllcc}
\mathbf{r}_{1}=\mathbf{2} \oplus \mathbf{1} & \mathbf{r}_{2}=\mathbf{2} & \mathbf{r}_{3}=\mathbf{1} \quad \mathbf{r}_{4}=1 & \mathbf{r}_{5}=\mathbf{2} \\
\mathbf{r}_{6}=\mathbf{2} \oplus \mathbf{1} & \mathbf{r}_{7}=\mathbf{3} \oplus \mathbf{1} \quad \mathbf{r}_{8}=\mathbf{3} \oplus \mathbf{2} & \mathbf{r}_{9}=\mathbf{4} \oplus \mathbf{2} \oplus \mathbf{2},
\end{array}
$$

where in particular the 8-forms come from $\hat{A}_{8,1}$ in Table 1 (doublet) and $\hat{A}_{10,1,1}$ in Table 2 (triplet), while the 9-forms come from $\hat{A}_{10,1,1}$ (quadruplet and first doublet) and $\hat{A}_{11,1}$ in Table 3 (second doublet). It is straightforward to verify that the dimensional reduction of the IIB fields of Table 4 gives rise to the same forms in 9 dimensions. In particular, the fields $A_{8}^{(\alpha \beta)}$ and $A_{9,1}^{\alpha}$ give rise to the triplet and the doublet of 8 -forms, while $A_{10}^{\alpha \beta \gamma}, A_{10}^{\alpha}$ and $A_{9,1}^{\alpha}$ give rise to the quadruplet and the two doublets of 9-forms.

One can check that eq. (3.1) holds, and that

$$
\mathbf{r}_{\mathbf{n}}=\mathbf{r}_{\mathbf{7 - n}}
$$




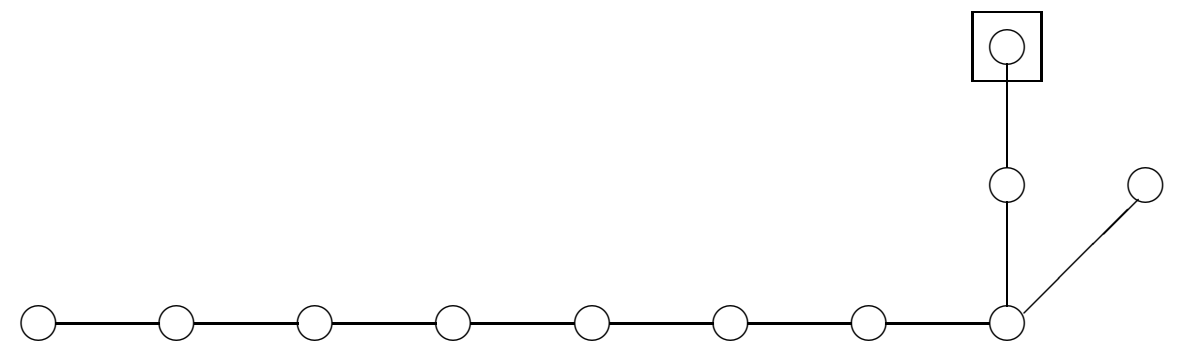

Figure 4: The $E_{11}$ Dynkin diagram corresponding to 9-dimensional supergravity. The non-abelian part of the internal symmetry group is $S L(2, \mathbb{R})$.

which is in agreement with eq. (3.2) since $S L(2, \mathbb{R})$ is pseudo-real. As already anticipated, the 7 -forms, dual to the scalars, belong to the adjoint representation. Finally, the spacetime-filling forms belong to the $\mathbf{4} \oplus \mathbf{2} \oplus \mathbf{2}$ of $S L(2, \mathbb{R})$. Like in IIB, the form associated to the spacetime-filling D-brane in 9 dimensions belongs to the quadruplet. In IIB it was shown that $\kappa$-symmetry of the effective action imposes a constraint on the charges, giving rise to a non-linear doublet of branes out of the quadruplet [50]. It would be interesting to see the same result occurring in 9 dimensions.

We now want to make contact with what is known about gauged supergravities in 9 dimensions. Using eq. (4.4), $E_{11}$ predicts that all the possible gauged maximal supergravities in 9 dimensions are generated by a mass parameter in the $\mathbf{3} \oplus \mathbf{2}$ of $S L(2, \mathbb{R})$. In [16] all the possible massive deformations of maximal 9-dimensional supergravities were classified. The analysis was performed taking into account not only the gauging of the symmetries of the 10-dimensional lagrangians, but also the gauging of the scaling symmetry of the equations of motions in 10 dimensions (so called "trombone" symmetries), as well as the dimensional reduction of the massive IIA theory obtained by gauging the scaling symmetry of the 11-dimensional field equations [53]. The result is that the independent mass parameters of 9-dimensional gauged supergravity belong to the $\mathbf{3} \oplus \mathbf{2} \oplus \mathbf{2} \oplus \mathbf{1}$ of $S L(2, \mathbb{R})$. The triplet can be seen as arising from the Scherk-Schwarz reduction of IIB, while one of the two doublets corresponds to the gauging of the $\mathbb{R}^{+}$symmetry of IIA (and its S-dual). The other doublet and the singlet arise from the gauging of the "trombone" symmetries, and as such they do not admit a lagrangian description. The outcome of our analysis is that the classification of gauged supergravities in terms of the $D-1$-forms arising from $E_{11}$ only accounts for the first two types of deformations, and we do not attempt here to provide an $E_{11}$ origin for the other massive theories. We will see that this result is completely general: 
in any dimension $D$, all the massive deformations of maximal supergravities which have a lagrangian description are in correspondence with the $D-1$-forms contained in $E_{11}$ in a $D$-dimensional background.

\section{$\mathrm{D}=8$}

The bosonic sector of maximal massless 8-dimensional supergravity [54] contains seven scalars parametrising the manifold $S L(3, \mathbb{R}) / S O(3) \times S L(2, \mathbb{R}) / S O(2)$, the metric, a vector in the $(\overline{\mathbf{3}}, \mathbf{2})$ of the internal symmetry group $S L(3, \mathbb{R}) \times S L(2, \mathbb{R})$, a 2 -form in $(\mathbf{3}, \mathbf{2})$ and a 3 -form which is a singlet of the internal symmetry group. The $E_{11}$ Dynkin diagram corresponding to this theory is shown in Fig. 5. From the diagram it is manifest why this is the first dimension for which there is a non-abelian enhancement in the internal symmetry.

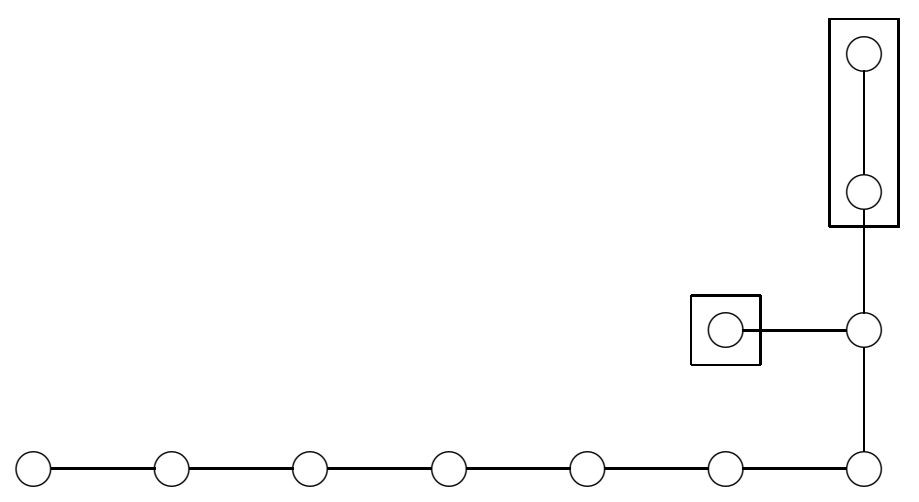

Figure 5: The $E_{11}$ Dynkin diagram corresponding to 8-dimensional supergravity. The internal symmetry group is $S L(3, \mathbb{R}) \times S L(2, \mathbb{R})$.

We now want to determine all the forms that $E_{11}$ predicts in 8 dimensions. As usual, this theory can be obtained from a dimensional reduction of either the 11-dimensional or the IIB theory. From 11 dimensions, there is a manifest $S L(3, \mathbb{R})$ symmetry arising, and therefore if one reduces the fields in Tables 1,2 and 3, the resulting forms are automatically collected in representations of this group. Similarly, from IIB the manifest symmetry is $S L(2, \mathbb{R}) \times S L(2, \mathbb{R})$, and the dimensional reduction of the fields in Table 4 indeed gives rise to forms carrying representations of this group in 8 dimensions. It turns out that from the IIB perspective, the $S L(2, \mathbb{R})$ group that gets enhanced to $S L(3, \mathbb{R})$ is the one corresponding to the internal symmetry of the ten dimensional theory. Using this, and 
the fact that the 8 dimensional theory is unique, one determines the representations of all the forms predicted by $E_{11}$ in 8 dimensions. We denote the representation carried by the $n$-form by $\mathbf{r}_{\mathbf{n}}$. The 1 -forms arise from the 11 -dimensional fields $\hat{g}^{1}{ }_{1}$ and $\hat{A}_{3}$ in Table 1 , giving two fields in the $\overline{\mathbf{3}}$ of $S L(3, \mathbb{R})$, or equivalently from the IIB fields $g^{1}{ }_{1}$ and $A_{2}^{\alpha}$ of Table 4 , giving a $(\mathbf{1}, \mathbf{2})$ and a $(\mathbf{2}, \mathbf{2})$ of $S L(2, \mathbb{R}) \times S L(2, \mathbb{R})$. The fact that the two dimensional reductions are the same implies $\mathbf{r}_{\mathbf{1}}=(\overline{\mathbf{3}}, \mathbf{2})$ of $S L(3, \mathbb{R}) \times S L(2, \mathbb{R})$. Using the same arguments, one obtains the representations of all the forms in the theory. In particular, the 7-forms arise from the 11-dimensional fields of Tables 1 and 2

$$
\hat{A}_{8,1} \rightarrow \mathbf{6} \oplus \overline{\mathbf{3}} \quad \hat{A}_{9,3} \rightarrow \overline{\mathbf{3}} \quad \hat{A}_{10,1,1} \rightarrow \mathbf{6}
$$

or from the IIB fields of Table 4

$$
\begin{aligned}
& A_{8}^{(\alpha \beta)} \rightarrow(\mathbf{3}, \mathbf{2}) \\
& A_{7,1} \rightarrow(\mathbf{1}, \mathbf{2})
\end{aligned} \quad A_{9,1}^{\alpha} \rightarrow(\mathbf{2}, \mathbf{2})
$$

where in eq. (4.6) the fields are representations of $S L(3, \mathbb{R})$ while in eq. (4.7) they are representations of $S L(2, \mathbb{R}) \times S L(2, \mathbb{R})$. This leads to $\mathbf{r}_{\mathbf{7}}=(\mathbf{6}, \mathbf{2}) \oplus(\overline{\mathbf{3}}, \mathbf{2})$ of $S L(3, \mathbb{R}) \times$ $S L(2, \mathbb{R})$. Similarly, the 8-forms arise from the 11-dimensional fields of Tables 1,2 and 3

$$
\begin{array}{lll}
\hat{A}_{8,1} \rightarrow \mathbf{3} & \hat{A}_{9,3} \rightarrow \mathbf{3} & \hat{A}_{10,1,1} \rightarrow \mathbf{3} \oplus \mathbf{1 5} \\
\hat{A}_{11,1} \rightarrow \mathbf{3} & \hat{A}_{11,3,1} \rightarrow \mathbf{3}
\end{array}
$$

or from the IIB fields of Table 4

$$
\begin{aligned}
& A_{8}^{(\alpha \beta)} \rightarrow(\mathbf{3}, \mathbf{1}) \quad A_{10}^{\alpha} \rightarrow(\mathbf{2}, \mathbf{1}) \quad A_{10}^{(\alpha \beta \gamma)} \rightarrow(\mathbf{4}, \mathbf{1}) \\
& A_{9,1}^{\alpha} \rightarrow(\mathbf{2}, \mathbf{3}) \oplus(\mathbf{2}, \mathbf{1}) \quad A_{8,2}^{\alpha} \rightarrow(\mathbf{2}, \mathbf{1}) \\
& A_{9,2,1} \rightarrow(\mathbf{1}, \mathbf{3}) \oplus(\mathbf{1}, \mathbf{1}) \quad A_{10,2}^{(\alpha \beta)} \rightarrow(\mathbf{3}, \mathbf{1}) \\
& A_{10,2} \rightarrow(\mathbf{1}, \mathbf{1}) \quad A_{10,2}^{\prime} \rightarrow(\mathbf{1}, \mathbf{1}) \quad A_{10,2,2}^{\alpha} \rightarrow(\mathbf{2}, \mathbf{1})
\end{aligned}
$$

and collecting the results in representations of $S L(3, \mathbb{R}) \times S L(2, \mathbb{R})$ this gives $\mathbf{r}_{\mathbf{8}}=(\mathbf{1 5}, \mathbf{1})$ $\oplus(\mathbf{3}, \mathbf{3}) \oplus(\mathbf{3}, \mathbf{1}) \oplus(\mathbf{3}, \mathbf{1})$. The final result is

$$
\begin{array}{ll}
\mathbf{r}_{1}=(\overline{3}, 2) & \mathbf{r}_{2}=(3,1) \quad \mathbf{r}_{3}=(1,2) \quad \mathbf{r}_{4}=(\overline{3}, \mathbf{1}) \\
\mathbf{r}_{5}=(3,2) & \mathbf{r}_{6}=(\mathbf{8}, \mathbf{1}) \oplus(\mathbf{1}, 3) \quad \mathbf{r}_{7}=(6,2) \oplus(\overline{3}, 2) \\
\mathbf{r}_{8}=(15,1) \oplus(3,3) \oplus(3,1) \oplus(3,1) &
\end{array}
$$


One can see that the spectrum is in perfect agreement with eq. (3.2), and therefore with the general rule that all the fields in $E_{11}$ satisfy duality relations. The doublet of 3-forms satisfies a self-duality relation, in agreement with the fact that a singlet of 3 -forms is present in the supergravity multiplet.

The 7-forms are associated to the massive deformations, that is to gauged maximal supergravities. Therefore, $E_{11}$ predicts that any massive deformation of 8-dimensional supergravity that admits a lagrangian description corresponds to a mass parameter in the $(\overline{\mathbf{6}}, \mathbf{2}) \oplus(\mathbf{3}, \mathbf{2})$. The most general gauged maximal supergravity theories in 8 dimensions were obtained in [17] using the Bianchi classification of group manifolds, but these results are not formulated in terms of representations of $S L(3, \mathbb{R}) \times S L(2, \mathbb{R})$. We believe that this classification is in agreement with $E_{11}$ once one considers the theories that admit a lagrangian description, like in nine dimensions. From eq. (4.9) one can see that the fields associated to the spacetime filling D-branes in 8 dimensions belong to the $(\mathbf{1 5}, \mathbf{1})$.

\section{$\mathrm{D}=7$}

The multiplet describing massless maximal supergravity theory in 7 dimensions [55] has a bosonic sector made of 14 scalars parametrising $S L(5, \mathbb{R}) / S O(5)$, the metric, a 1 -form in the $\overline{\mathbf{1 0}}$ and a 2 -form in the $\mathbf{5}$ of $S L(5, \mathbb{R})$. The $E_{11}$ Dynkin diagram corresponding to the 7-dimensional theory is shown in Fig. 6. One can see from the diagram that the 1-forms carry two antisymmetric indices of $S L(5, \mathbb{R})$.

The analysis of all the forms that result from the dimensional reduction of the fields in Tables 1,2 and 3 proceeds like in the cases already considered, taking care of the fact that the forms naturally arise as representations of $S L(4, \mathbb{R})$. Since the internal symmetry group is $S L(5, \mathbb{R})$, the individual $S L(4, \mathbb{R})$ representations of a given form must collect up into $S L(5, \mathbb{R})$ representations. Starting from the 1-form, one has, in terms of representations of $S L(4, \mathbb{R})$,

$$
\hat{g}_{1}^{1} \rightarrow \overline{4} \quad \hat{A}_{3} \rightarrow \mathbf{6}
$$

and $\overline{\mathbf{4}} \oplus \mathbf{6}=\overline{\mathbf{1 0}}$ of $S L(5, \mathbb{R})$. Similarly, the 2 -forms come from

$$
\hat{A}_{3} \rightarrow 4 \quad \hat{A}_{6} \rightarrow \mathbf{1}
$$

and $\mathbf{4} \oplus \mathbf{1}=\mathbf{5}$. Proceeding this way, one obtains all the representations of the propagating forms from the fields in Table 1 . In particular, the 5-forms arise from

$$
\hat{A}_{6} \rightarrow \mathbf{4} \quad \hat{A}_{8,1} \rightarrow \mathbf{1 5} \oplus \mathbf{1} \quad \hat{A}_{9,3} \rightarrow \overline{\mathbf{4}}
$$




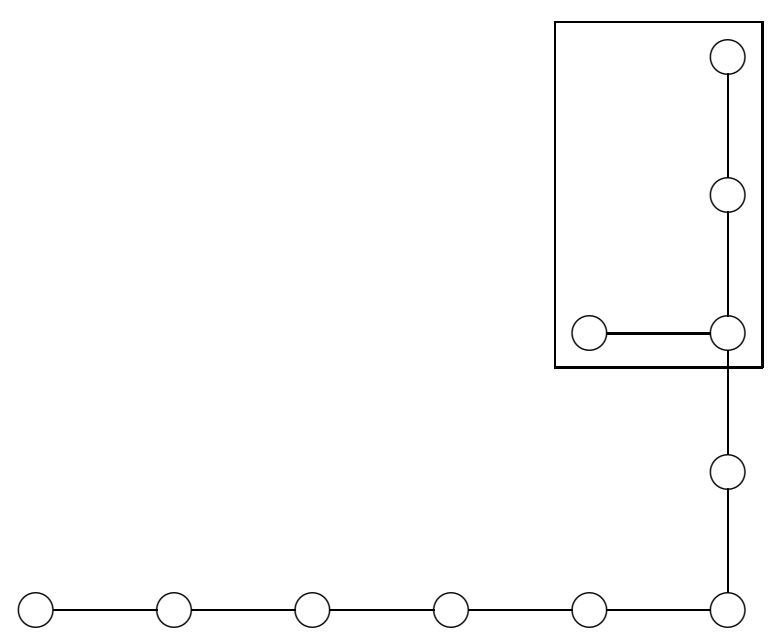

Figure 6: The $E_{11}$ Dynkin diagram corresponding to 7-dimensional supergravity. The internal symmetry group is $S L(5, \mathbb{R})$.

and $\mathbf{1} \oplus \mathbf{4} \oplus \overline{\mathbf{4}} \oplus \mathbf{1 5}=\mathbf{2 4}$, which is the adjoint of $S L(5, \mathbb{R})$. We now determine the 6-forms, resulting from the fields in Tables 1 and 2 . The result is

$$
\begin{aligned}
& \hat{A}_{6} \rightarrow \mathbf{1} \quad \hat{A}_{8,1} \rightarrow \overline{\mathbf{4}} \oplus \overline{\mathbf{2 0}} \\
& \hat{A}_{9,3} \rightarrow \mathbf{6} \oplus \overline{\mathbf{1 0}} \quad \hat{A}_{10,1,1} \rightarrow \mathbf{1 0} \quad \hat{A}_{10,4,1} \rightarrow \mathbf{4}
\end{aligned}
$$

and since $\mathbf{1} \oplus \overline{\mathbf{4}} \oplus \overline{\mathbf{1 0}}=\overline{\mathbf{1 5}}$ and $\mathbf{4} \oplus \mathbf{6} \oplus \mathbf{1 0} \oplus \overline{\mathbf{2 0}}=\overline{\mathbf{4 0}}$, this implies $\mathbf{r}_{\mathbf{6}}=\overline{\mathbf{1 5}} \oplus \overline{\mathbf{4 0}}$. Finally, we determine the 7-forms, resulting from the fields in Tables 1, 2 and 3 . We have

$$
\begin{aligned}
& \hat{A}_{8,1} \rightarrow \mathbf{6} \oplus \mathbf{1 0} \quad \hat{A}_{9,3} \rightarrow \mathbf{4} \oplus \mathbf{2 0} \\
& \hat{A}_{10,1,1} \rightarrow \mathbf{4} \oplus \mathbf{3 6} \quad \hat{A}_{10,4,1} \rightarrow \mathbf{1} \oplus \mathbf{1 5} \\
& \hat{A}_{11,1} \rightarrow \mathbf{4} \quad \hat{A}_{11,3,1} \rightarrow \mathbf{1 5} \quad \hat{A}_{11,4} \rightarrow \mathbf{1} \quad \hat{A}_{11,4,3} \rightarrow \overline{\mathbf{4}}
\end{aligned}
$$

which in terms of representations of $S L(5, \mathbb{R})$ becomes $\mathbf{r}_{\mathbf{7}}=\mathbf{5} \oplus \mathbf{4 5} \oplus \mathbf{7 0}$.

The complete result is

$$
\begin{aligned}
& r_{1}=\overline{\mathbf{1 0}} \quad r_{2}=5 \quad r_{3}=\overline{5} \quad r_{4}=10 \quad r_{5}=24 \\
& r_{6}=\overline{\mathbf{1 5}} \oplus \overline{\mathbf{4 0}} \quad r_{7}=5 \oplus 45 \oplus 70
\end{aligned}
$$

The fact that all the possible gaugings of 7-dimensional maximal supergravity are encoded in a mass parameter belonging to the $\mathbf{1 5} \oplus \mathbf{4 0}$ of $S L(5, \mathbb{R})$ was shown in [18]. Given 
that the masses are related by dualities to the field strengths of the 6-forms, they should indeed belong to the representation $\mathbf{r}_{\mathbf{6}}^{*}$, and this is in complete agreement with our results.

The well-known $S O(5)$-gauged maximal supergravity theory in 7 dimensions [56], corresponding to 11-dimensional supergravity compactified on $S^{4}$, is a particular case of the class of theories corresponding to a mass parameter which is a singlet of $S O(5)$. To find such a singlet, one looks at the particular form of the representations contained in $\mathbf{r}_{\mathbf{6}}$ and one realises that it can only come from the $\mathbf{1 5}$, which is a symmetric second rank tensor [18]. From eq. (4.14) it turns out that the $S O(5)$ singlet in the $\mathbf{1 5}$ can only arise from the

fields $\hat{A}_{6}$ and $\hat{A}_{9,3}$, which are (duals of) supergravity fields. By contrast, the fields $\hat{A}_{10,1,1}$ and $\hat{A}_{10,4,1}$, which are not traditional supergravity fields in 11 dimensions, can only give rise to massive deformations in the $\mathbf{4 0 .}$

\section{$\mathrm{D}=6$}

We now consider the six-dimensional case. The symmetry of the massless maximal supergravity theory in 6 dimensions $[57]$ is $S O(5,5)$, and the bosonic sector of the theory describes 25 scalars parametrising $S O(5,5) /[S O(5) \times S O(5)]$, the metric, a 1-form in the 16 and a 2 -form in the $\mathbf{1 0}$, whose field strength satisfies a self-duality condition. The $E_{11}$ Dynkin diagram corresponding to the 6-dimensional background in shown in Fig. 7. From the diagram it is manifest that the 1 -forms belong to the spinor representation.

We repeat here the same analysis that was performed in the higher dimensional cases, listing all the forms arising from the dimensional reduction as representations of $S L(5, \mathbb{R})$, and then showing that for each form these representations collect in representations of $S O(5,5)$. The 1-forms arise from the first three fields in Table 1,

$$
\hat{g}^{1}{ }_{1} \rightarrow \overline{\mathbf{5}} \quad \hat{A}_{3} \rightarrow \mathbf{1 0} \quad \hat{A}_{6} \rightarrow \mathbf{1},
$$

which results in $\mathbf{1 0} \oplus \overline{\mathbf{5}} \oplus \mathbf{1}=\mathbf{1 6}=\mathbf{r}_{\mathbf{1}}$, following [47]. The 2-forms arise from

$$
\hat{A}_{3} \rightarrow \mathbf{5} \quad \hat{A}_{6} \rightarrow \overline{\mathbf{5}}
$$

and $\mathbf{5} \oplus \overline{\mathbf{5}}=\mathbf{1 0}=\mathbf{r}_{\mathbf{2}}$. The 3 -forms dual to the vectors are generated from

$$
\hat{A}_{3} \rightarrow \mathbf{1} \quad \hat{A}_{6} \rightarrow \overline{\mathbf{1 0}} \quad \hat{A}_{8,1} \rightarrow \mathbf{5},
$$

thus giving $\mathbf{r}_{\mathbf{3}}=\overline{\mathbf{1 6}}$. The 4 -forms, dual to the scalars, arise from

$$
\hat{A}_{6} \rightarrow \mathbf{1 0} \quad \hat{A}_{8,1} \rightarrow \mathbf{2 4} \oplus \mathbf{1} \quad \hat{A}_{9,3} \rightarrow \overline{\mathbf{1 0}},
$$




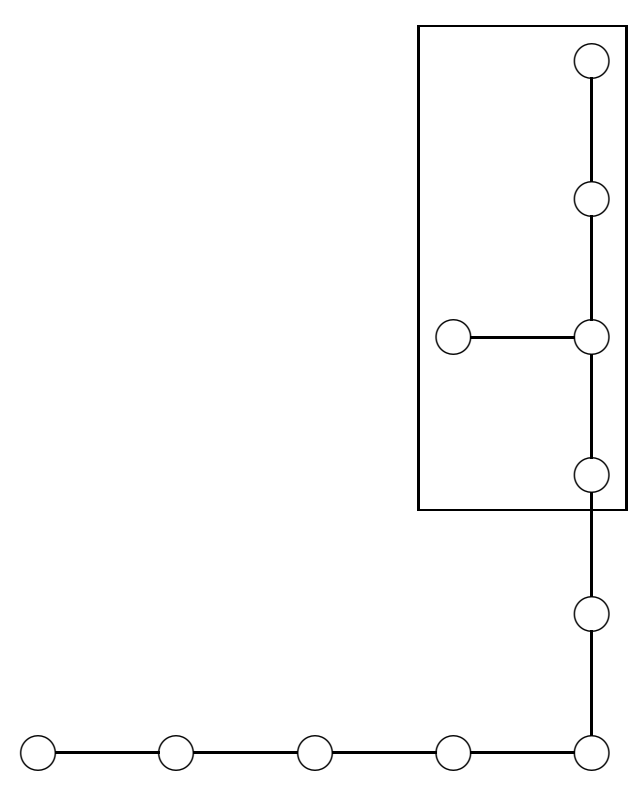

Figure 7: The $E_{11}$ Dynkin diagram corresponding to 6-dimensional supergravity. The internal symmetry group is $S O(5,5)$.

which results in $\mathbf{r}_{\mathbf{4}}=\mathbf{4 5}$, which is the adjoint of $S O(5,5)$.

The 5-forms whose field strengths are dual to masses, originate form the same 11dimensional fields as did the 4-forms, giving in this case the representations

$$
\hat{A}_{6} \rightarrow \mathbf{5} \quad \hat{A}_{8,1} \rightarrow \overline{\mathbf{5}} \oplus \overline{\mathbf{4 5}} \quad \hat{A}_{9,3} \rightarrow \mathbf{1 0} \oplus \mathbf{4 0}
$$

as well as from the first two fields in Table 2, giving the representations

$$
\hat{A}_{10,1,1} \rightarrow \mathbf{1 5} \quad \hat{A}_{10,4,1} \rightarrow \mathbf{2 4}
$$

Summing up all the representations of eqs. (4.21) and (4.22) one gets $\mathbf{r}_{\mathbf{5}}=\mathbf{1 4 4}$ of $S O(5,5)$. Finally, we consider the 6-forms. These arise from the fields in eqs. (4.21) and (4.22), giving rise to the representations

$$
1 \quad \overline{\mathbf{1 0}} \oplus \overline{\mathbf{4 0}} \quad \mathbf{5} \oplus \mathbf{4 5} \oplus \mathbf{5 0} \quad \mathbf{5} \oplus \mathbf{7 0} \quad \overline{\mathbf{5}} \oplus \overline{\mathbf{4 5}} \oplus \overline{\mathbf{7 0}}
$$

respectively, as well as from the first five fields in Table 3, giving rise to the representations

$$
\begin{array}{lllll}
5 & \overline{45} & \overline{5} & 40 & 15
\end{array},
$$

and summing all up one gets $\mathbf{r}_{\mathbf{6}}=\mathbf{1 0} \oplus \overline{\mathbf{1 2 6}} \oplus \mathbf{3 2 0}$. 
Summarising, we get

$$
\begin{array}{llll}
r_{1}=16 & r_{2}=10 & r_{3}=\overline{16} & r_{4}=45 \\
r_{5}=144 & r_{6}=10 \oplus \overline{126} \oplus 320
\end{array}
$$

The fact that the most general gauged maximal six-dimensional supergravity arises from a mass deformation belonging to the $\overline{\mathbf{1 4 4}}$ of $S O(5,5)$ has been shown in [19]. Our results are therefore in complete agreement with the literature.

\section{$\mathrm{D}=5$}

We now consider the five-dimensional case. One can see from Fig. 8 the appearance of the exceptional group $E_{6(+6)}$, which is the maximally non-compact version of $E_{6}$.

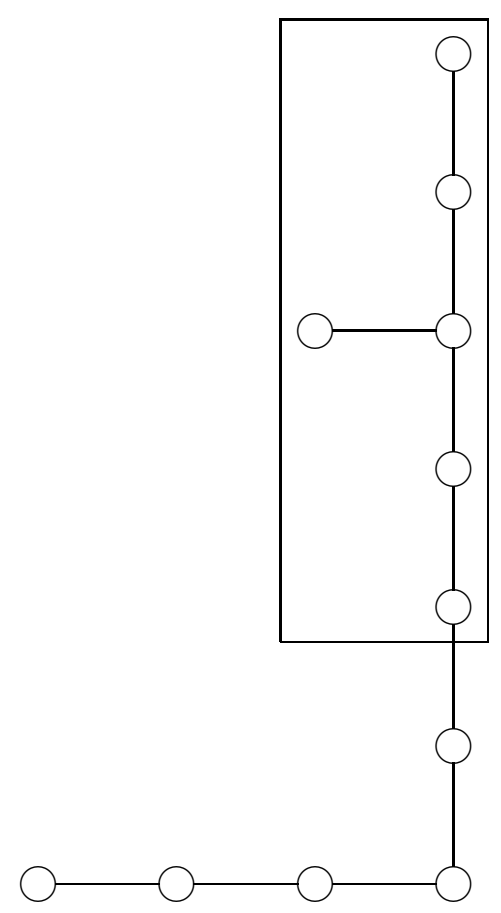

Figure 8: The $E_{11}$ Dynkin diagram corresponding to 5-dimensional supergravity. The internal symmetry group is $E_{6(+6)}$.

The bosonic sector of maximal massless supergravity theory in five dimensions $[7,15]$ contains 42 scalars parametrising the manifold $E_{6(+6)} / U S p(8)$, the metric and a 1-form in the 27. These fields and their duals result from the dimensional reduction of the first six 
fields in Table 1. In the dimensional reduction, the fields naturally arise as representations of $S L(6, \mathbb{R})$, and using the decomposition rules of [47] one can see that for each form the representations collect in representations of $E_{6}$. The 1 -forms come from $\hat{g}_{1}^{1}, \hat{A}_{3}$ and $\hat{A}_{6}$, giving the representations

$$
\overline{6} \quad 15 \quad \overline{6},
$$

thus resulting in $\mathbf{r}_{\mathbf{1}}=\mathbf{2 7}$ of $E_{6}$. Their dual 2-forms come from $\hat{A}_{3}, \hat{A}_{6}$ and $\hat{A}_{8,1}$, giving

$$
6 \overline{15} \quad 6,
$$

which gives $\mathbf{r}_{2}=\overline{\mathbf{2 7}}$. The 3 -forms dual to the scalars arise from the $\hat{A}_{3}, \hat{A}_{6}, \hat{A}_{8,1}, \hat{A}_{9,3}$ and $\hat{A}_{9,6}$, and give

$$
\begin{array}{lllll}
1 & 20 & 1 \oplus 35 & 20 & 1
\end{array}
$$

respectively. This corresponds to the $\mathbf{7 8}$, which is the adjoint of $E_{6}$.

We now consider the 4 -forms, whose field strengths are dual to masses. They arise from the fields $\hat{A}_{6}, \hat{A}_{8,1}, \hat{A}_{9,3}$ and $\hat{A}_{9,6}$ in Table 1 , as well as from the first three fields in Table 2. The Table 1 fields give the representations

$$
15 \quad \overline{6} \oplus \overline{84} \quad 15 \oplus 105 \quad \overline{6},
$$

while those in Table 2 give

$$
21 \quad \overline{84} \quad 15 .
$$

The overall sum gives $\mathbf{r}_{4}=\mathbf{3 5 1}$ of $E_{6}$.

Finally, we analyse the 5-forms. These arise from the same 11-dimensional fields as did the 4-forms, giving respectively the representations

$$
\begin{array}{lrrr}
6 & \overline{\mathbf{1 5}} \oplus \overline{\mathbf{1 0 5}} \quad 6 \oplus \mathbf{6 4} \oplus \mathbf{2 1 0} \quad \overline{\mathbf{1 5}} \\
6 \oplus \mathbf{1 2 0} \quad \overline{\mathbf{1 5}} \oplus \overline{\mathbf{1 0 5}} \oplus \overline{\mathbf{3 8 4}} \quad 6 \oplus \mathbf{8 4},
\end{array}
$$

as well as from the first nine fields in Table 3, giving

$$
\begin{array}{llllll}
6 & \overline{105} & \overline{15} & 210 & 120 \\
6 \oplus 6 & \overline{105} & \overline{15} & 6
\end{array}
$$

This results in $\mathbf{r}_{\mathbf{5}}=\overline{\mathbf{2 7}} \oplus \overline{\mathbf{1 7 2 8}}$. Observe that the fact that the sixth field in Table 3 has multiplicity 2 is crucial in order to collect the fields in representations of $E_{6}$. Summarising 
the results, we have found

$$
\begin{array}{ll}
\mathrm{r}_{1}=27 & \mathrm{r}_{2}=\overline{\mathbf{2 7}} \quad \mathrm{r}_{3}=78 \\
\mathrm{r}_{4}=351 & \mathrm{r}_{5}=\overline{\mathbf{2 7}} \oplus \overline{\mathbf{1 7 2 8}}
\end{array}
$$

The fact that the most general five-dimensional gauged maximal supergravity results from a mass deformation in the $\overline{\mathbf{3 5 1}}$ of $E_{6}$ has been shown in [20]. This contains the case in which the symmetry $S O(6)$ is gauged [14], which corresponds to the reduction of IIB on $S^{5}$ [49]. The resulting $A d S_{5} \times S^{5}$ background is the near-horizon geometry of the solution corresponding to a stack of D3-branes, and this theory has received a lot of attention in the context of the AdS/CFT correspondence. The corresponding supergravity arises from an $S O(6)$ singlet in the $\mathbf{3 5 1}$ deformation. This singlet arises from the 11-dimensional field $\hat{A}_{10,1,1}$ that contains the $\mathbf{2 1}$ of $S L(6, \mathbb{R})$, which is a symmetric second rank tensor. This field is not a traditional 11-dimensional supergravity field and correspondingly the theory does not arise from a compactification of 11-dimensional supergravity. On the other hand, one can show that the 4 -forms arising from the dimensional reduction of the IIB fields are such that the mass parameter which is a singlet of $S O(6)$ corresponds to the compactification of the traditional supergravity fields in IIB, corresponding to the fact that the five-dimensional theory has a geometric origin from the perspective of IIB supergravity. In the next section, when we will show how the gauging originates from the $E_{11}$ non-linear realisation, we will concentrate in particular on the five-dimensional case.

\section{$\mathrm{D}=4$}

The global symmetry of four-dimensional massless maximal supergravity is $E_{7(+7)}$, which is the maximally non-compact version of $E_{7}$. This symmetry rotates electric and magnetic vectors, and as such it is not a symmetry of the lagrangian, but only of the equations of motion. This is in agreement with $E_{11}$, in which fields and their magnetic duals are treated on the same footing, and indeed Fig. 9 shows that the symmetry $E_{7}$ arises naturally in a four-dimensional background of $E_{11}$.

The bosonic field content of the supergravity theory contains 70 scalars parametrising the manifold $E_{7(+7)} / S U(8)$, the metric and 28 vectors, that together with their magnetic duals make the 56 of $E_{7}$. We now classify all the forms arising from the dimensional reduction of the fields in Tables 1,2 and 3 . The resulting forms will be representations of $S L(7, \mathbb{R})$, and as a consequence of the symmetry enhancement, for each form these 


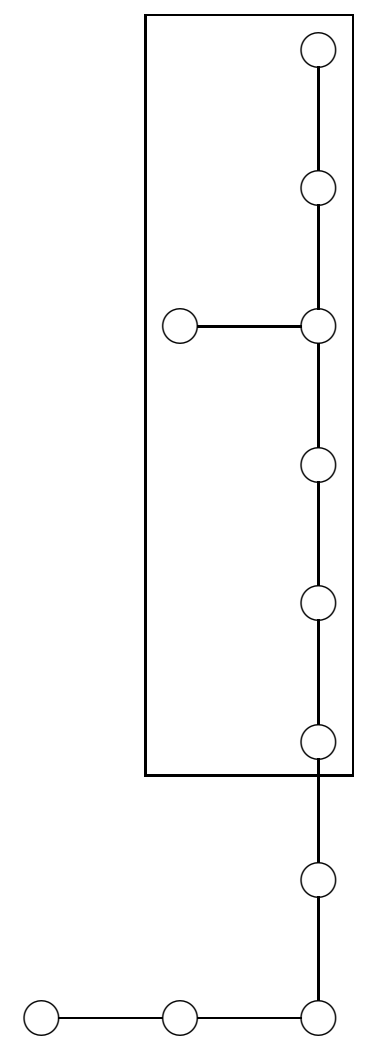

Figure 9: The $E_{11}$ Dynkin diagram corresponding to 4-dimensional supergravity. The internal symmetry group is $E_{7(+7)}$.

representations will collect to representations of $E_{7}$. The 1-forms arise from $\hat{g}^{1}{ }_{1}, \hat{A}_{3}, \hat{A}_{6}$ and $\hat{A}_{8,1}$ in Table 1, giving the representations

$$
\overline{7} \quad 21 \quad \overline{21} \quad 7,
$$

whose sum is the $\mathbf{5 6}$ of $E_{7}$. The 2-forms are dual to the scalars, and arise from the fields $\hat{A}_{3}, \hat{A}_{6}, \hat{A}_{8,1}, \hat{A}_{9,3}$ and $\hat{A}_{9,6}$ in Table 1, resulting in

$$
\begin{array}{lllll}
7 & \overline{35} & 48 \oplus 1 & 35 & \overline{7}
\end{array},
$$

which is the 133 , that is the adjoint of $E_{7}$.

We now consider the 3 -forms. The fields contributing from Table 1 are the same that generate the 2-forms, giving the representations

$$
1 \quad 35 \quad \overline{7} \oplus 140 \quad 21 \oplus \overline{\mathbf{2 2 4}} \quad \overline{\mathbf{2 1}} \oplus \overline{\mathbf{2 8}}
$$


as well as the first six fields in Table 2, giving

$$
\begin{array}{llllll}
28 & 224 & \overline{140} & 7 & \overline{35} & 1
\end{array} .
$$

The collection of these representations is the 912 of $E_{7}$.

Finally, we consider the 4 -forms. These arise from the fields $\hat{A}_{6}, \hat{A}_{8,1}, \hat{A}_{9,3}$ and $\hat{A}_{9,6}$ in Table 1, plus the first six fields in Table 2 and all the fields in Table 3. The fields in Table 1 give the representations

$$
21 \quad \overline{21} \oplus 224 \quad 7 \oplus \overline{\mathbf{1 4 0}} \oplus 588 \quad \overline{\mathbf{3 5}} \oplus \overline{\mathbf{1 1 2}},
$$

while the fields in Table 2 give

$$
7 \oplus 189 \quad \overline{\mathbf{5 5}} \oplus \mathbf{2 1 0} \oplus \mathbf{1 3 2 3} \quad 48 \oplus 392 \oplus \overline{\mathbf{5 4 0}} \quad \mathbf{1} \oplus 48 \quad 35 \oplus \overline{\mathbf{2 1 0}} \quad \overline{7}
$$

and the fields in Table 3 give

\begin{tabular}{lcccccc}
7 & 210 & $\overline{35}$ & 784 & \multicolumn{2}{c}{540} & \multicolumn{2}{c}{$48 \oplus 48$} \\
$\overline{1323}$ & $\overline{210}$ & $\overline{189}$ & 1 & 112 & $35 \oplus 35$ \\
$\overline{588}$ & 140 & $\overline{7} \oplus \overline{7}$ & $\overline{224}$ & 21 & $\overline{21}$
\end{tabular}

The sum of all these representations gives $\mathbf{r}_{\mathbf{4}}=\mathbf{1 3 3} \oplus \mathbf{8 6 4 5}$. Like in the five-dimensional case, it is crucial that some fields in Table 3 have multiplicity 2 in order for the 4 -forms to collect in representations of $E_{7}$. Summarising,

$$
\mathrm{r}_{1}=56 \quad \mathrm{r}_{2}=133 \quad \mathrm{r}_{3}=912 \quad \mathrm{r}_{4}=133 \oplus 8645 .
$$

In [19] it has been shown that the most general four-dimensional gauged supergravity corresponds to a mass deformation in the $\mathbf{9 1 2}$ of $E_{7}$. In order to recover all the known gaugings from this $E_{7}$ covariant approach, it was crucial to use a formulation of the theory in which the vectors and their magnetic duals were treated on the same footing [21]. This is in complete agreement with our results. A well-known example is the $S O(8)$-gauged maximal supergravity theory [12] corresponding to 11-dimensional supergravity compactified on $S^{7}$. In order to see which mass parameter one has to turn on in order to gauge an $S O(8)$ subgroup of $E_{7}$, one first has to collect the $S L(7, \mathbb{R})$ representations of the 3 -forms in representations of $S L(8, \mathbb{R})$. This is achieved using

$$
36=28 \oplus 7 \oplus 1
$$


and

$$
420=224 \oplus 140 \oplus \overline{35} \oplus \overline{\mathbf{2 1}}
$$

In terms of representations of $S L(8, \mathbb{R})$, the $\mathbf{9 1 2}$ of $E_{7}$ decomposes as

$$
912=420 \oplus \overline{420} \oplus 36 \oplus \overline{36}
$$

There are two $S O(8)$ singlets, one in the $\mathbf{3 6}$ and one in the $\overline{\mathbf{3 6}}$. The one in the $\overline{\mathbf{3 6}}$ arises from the fields $\hat{A}_{9,6}$ and $\hat{A}_{3}$, as can be seen from eq. (4.36), which are (dual of) supergravity fields, in agreement with the 11-dimensional supergravity origin of the four-dimensional theory. The singlet in the $\mathbf{3 6}$ arises from the fields $\hat{A}_{10,1,1}$ and $\hat{A}_{10,7,7}$, showing the presence of a fourdimensional gauged $S O(8)$ theory with no 11-dimensional supergravity origin. This agrees with the fact that $E_{7}$ maps electric fields to magnetic fields, and therefore is not a symmetry of the lagrangian, but only of the equations of motion. The electromagnetic duality of the four dimensional theory, corresponding to the IIA/IIB T-duality, maps representations of $S L(8, \mathbb{R})$ to their complex conjugates. Therefore, the gauged $S O(8)$ theory that does not seem to have an 11-dimensional geometric origin corresponds to the gauging of the magnetic fields.

\section{$\mathrm{D}=3$}

We finally consider the three-dimensional case. The bosonic sector of massless maximal supergravity theory in three dimensions [58] describes 128 scalars parametrising the manifold $E_{8} / S O(16)$ and the metric. We expect that the 1-forms are in the $\mathbf{2 4 8}$ of $E_{8}$, according to the general rule for which the fields dual to the scalars are in the adjoint representation. The fact that $E_{11}$ predicts an $E_{8}$ symmetry in a three-dimensional background, as well as the representation carried by the 1-forms, is transparent from the Dynkin diagram in Fig. 10.

We now determine all the 1-forms and 2-forms coming from the dimensional reduction of the fields in Tables 1 and 2. These forms naturally carry representations of $S L(8, \mathbb{R})$, and as a result of the symmetry enhancement these representations can be grouped in representations of $E_{8}$. The 1-forms arise from all the fields in Table 1, giving the representations

$$
\overline{8} \quad 28 \quad \overline{56} \quad 63 \oplus 1 \quad 56 \quad \overline{28} \quad 8 \quad,
$$

thus resulting in $\mathbf{r}_{1}=\mathbf{2 4 8}$ that is the adjoint of $E_{8}$, as already anticipated. The 2 -forms come from all the fields in Table 1 with the exception of the metric, as well as from all the 


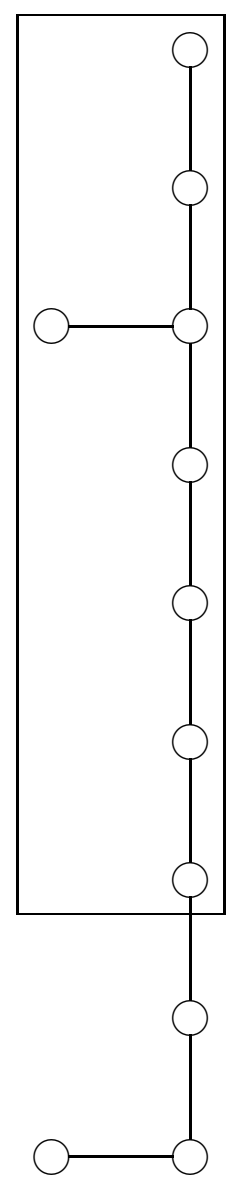

Figure 10: The $E_{11}$ Dynkin diagram corresponding to 3-dimensional supergravity. The internal symmetry group is $E_{8(+8)}$.

fields in Table 2. The fields in Table 1 generate the representations

$$
8 \quad 70 \quad 216 \oplus \overline{8} \quad \overline{420} \oplus 28 \quad \overline{168} \oplus \overline{56} \quad 63 \oplus 1
$$

while the fields in Table 2 generate

$\begin{array}{llrrr}36 & 504 & 720 & 63 & \overline{504} \\ \overline{36} & 1 & 168 & 56 & 420 \\ \overline{28} & \overline{216} & 8 & 70 & \overline{8}\end{array}$

All these representations collect in $\mathbf{r}_{2}=\mathbf{1} \oplus \mathbf{3 8 7 5}$ of $E_{8}$. Summarising, we have found that

$$
\mathrm{r}_{1}=248 \quad \mathrm{r}_{2}=1 \oplus 3875
$$


We leave the determination of the 3 -forms in three dimensions as an open project. Following eq. (3.1) we expect these to be contained in

$$
\mathbf{r}_{1} \otimes \mathrm{r}_{2}=\mathbf{2 4 8} \oplus \mathbf{2 4 8} \oplus \mathbf{3 8 7 5} \oplus \mathbf{3 0 3 8 0} \oplus \mathbf{1 4 7 2 5 0 \oplus 7 7 9 2 4 7} .
$$

Since the vectors are dual to the scalars in three dimensions, the study of gauged supergravities requires a formulation in which one dualises scalars into vectors. In has been shown in [22] that the most general gauging arises from a mass deformation in the $1 \oplus \mathbf{3 8 7 5}$ of $E_{8}$, and this is once again in agreement with the $E_{11}$ results.

To conclude this section, we stress that all gauged maximal supergravities possess an 11dimensional origin within the $E_{11}$ formulation of the low-energy action of M-theory, which also provides a prediction for all the spacetime-filling forms allowed in the supersymmetry algebras of such supergravities in any dimension.

\section{Gauging of maximal supergravities as a non-linear realisation}

In the previous sections we have classified all possible massive supergravities using $E_{11}$ and at the same time provided a common framework for all such theories. In this section we will show, in outline, how the $E_{11}$ non-linear realisation also encodes the dynamics of such theories and, in particular, we will see how the phenomenon of gauging arises in massive supergravities. We will concentrate on the five-dimensional case, but our analysis can be readily generalised to all the other theories. The procedure is similar to the one carried out in [29], where it was shown that the massive IIA theory of Romans results from a non-linear realisation. All the gauged supergravities in five dimensions were classified in [20] and to some extent we will use similar notation in order to facilitate the comparison of the formulae. However, the latter reference used the supersymmetry of the theory and so the derivation of their results has little in common with that given here.

The five dimensional massless theory has an internal $E_{6}$ symmetry and in its $E_{11}$ for-

mulation the generators corresponding to the forms with the exception of the space-filling 5 -forms are given by

$$
R^{\alpha} \quad R^{a, M} \quad R_{M}^{a b} \quad R^{a b c, \alpha} \quad R_{[M N]}^{a b c d},
$$

where $R^{\alpha}, \alpha=1, \ldots, 78$ are the $E_{6}$ generators, and an upstairs $M$ index, $M=1, \ldots, 27$, corresponds to the $\overline{\mathbf{2 7}}$ representation, a downstairs $M$ index to the $\mathbf{2 7}$ and a pair of anti- 
symmetric downstairs indices $[M N]$ correspond to the $\overline{\mathbf{3 5 1}}$ as the tenor product of $\mathbf{2 7} \otimes \mathbf{2 7}$ in the anti-symmetric combination only contains the $\overline{\mathbf{3 5 1}}$. We write the commutation relations for the $E_{6}$ generators in the form

$$
\left[R^{\alpha}, R^{\beta}\right]=f_{\gamma}^{\alpha \beta} R^{\gamma}
$$

where $f^{\alpha \beta}$ are the structure constants of $E_{6}$. The commutator of these generators with the 1 -form is determined by the fact that the Jacobi identity involving $R^{\alpha}, R^{\beta}$ and $R^{a, M}$ demands that this generator is in a representation of $E_{6}$, which is in fact the $\overline{\mathbf{2 7}}$ as noted above, and it is given by

$$
\left[R^{\alpha}, R^{a, M}\right]=\left(D^{\alpha}\right)_{N}{ }^{M} R^{a, N},
$$

where $\left(D^{\alpha}\right)_{N}{ }^{M}$ are the generators of $E_{6}$ in this representation and so obey

$$
\left[D^{\alpha}, D^{\beta}\right]_{M}^{N}=f_{\gamma}^{\alpha \beta}\left(D^{\gamma}\right)_{M}^{N} .
$$

The two form generators are in the $\mathbf{2 7}$ representation and so their commutator with the generators of $E_{6}$ is given by

$$
\left[R^{\alpha}, R^{a b}{ }_{M}\right]=-\left(D^{\alpha}\right)_{M}{ }^{N} R^{a b}{ }_{N}
$$

This involves the matrix $\left(D^{\alpha}\right)_{M}{ }^{N}$ in the way that follows from the fact that if we contract the indices of a $\overline{\mathbf{2 7}}$ with a $\mathbf{2 7}$ we find an $E_{6}$ invariant. The $E_{6}$ commutator of the $R^{a b c, \alpha}$ is given by

$$
\left[R^{\alpha}, R^{a b c, \beta}\right]=f_{\gamma}^{\alpha \beta} R^{a b c, \gamma} .
$$

as it is in the adjoint representation while that of the $R^{a b c d}{ }_{[M N]}$ generator is given by

$$
\left[R^{\alpha}, R_{[M N]}^{a b c d}{ }_{[M]}=-\left(D^{\alpha}\right)_{M}^{P} R_{[P N]}^{a b c d}-\left(D^{\alpha}\right)_{N}^{P} R_{[M P]}^{a b c d}{ }_{[M}\right.
$$

The next commutators of the $E_{11}$ algebra to consider are those of the 1-forms which yield a 2-form and are given by

$$
\left[R^{a, M}, R^{b, N}\right]=d^{M N P} R_{P}^{a b},
$$

where $d^{M N P}$ is required by the Jacobi identity involving $R^{\alpha}, R^{a, M}$ and $R^{b, N}$ to be an invariant tensor transforming in the $\overline{\mathbf{2 7}} \otimes \overline{\mathbf{2 7}} \otimes \overline{\mathbf{2 7}}$ representation and so it is also a symmetric tensor. The commutator of a 1 -form with a 2 -form generator is a 3 -form generator and 
the Jacobi identities involving $R^{\alpha}, R^{a, N}$ and $R^{b c}{ }_{M}$ imply that this is given in terms of the $\left(D^{\alpha}\right)_{M^{N}}$ matrix as follows:

$$
\left[R^{a, N}, R_{M}^{b c}\right]=g_{\alpha \beta}\left(D^{\alpha}\right)_{M}^{N} R^{a b c, \beta}
$$

where $g_{\alpha \beta}$ is the Killing metric. As mentioned above the 4-form generator is in the $\overline{\mathbf{3 5 1}}$ representation and as this is the only representation in the anti-symmetric tenor product of $\mathbf{2 7} \otimes \mathbf{2 7}$ it appears on the right-hand side of the commutators of two 2 -forms as

$$
\left[R_{M}^{a b}, R_{N}^{c d}\right]=R_{[M N]}^{a b c d},
$$

as well as in the commutator of the 1 -form with the 3 -form,

$$
\left[R^{a, M}, R^{b c d, \alpha}\right]=S^{\alpha M[N P]} R_{[N P]}^{a b c d},
$$

where $S^{\alpha M[N P]}$ is an invariant tensor. Using

$$
g_{\beta \gamma}\left(D^{\alpha}\right)_{M}^{N}\left(D^{\gamma}\right)_{N}^{M}=k \delta_{\beta}^{\alpha}
$$

one can show that the Jacobi identities constrain the invariant tensor $S^{\alpha M[N P]}$ to satisfy

$$
S^{\alpha M[N P]}+\frac{1}{k} g_{\beta \gamma}\left(D^{\alpha} D^{\beta}\right)_{Q}{ }^{M} S^{\gamma Q[N P]}=-\frac{1}{k}\left(D^{\alpha}\right)_{Q}{ }^{[N} d^{P] M Q} .
$$

One can show that the Jacobi identities are also compatible with a 5 -form generator in the $\mathbf{2 7} \oplus \mathbf{1 7 2 8}$, in agreement with the results of the previous section.

We now want to show how the massive supergravities arise as a non-linear realisation. The massless maximal five dimensional supergravity is the non-linear realisation of the above algebra once we include the space-time translations $P_{a}$. We will follow the derivation given in [29] for the case of Romans theory. In order to account for the massive deformations we now adopt the commutator

$$
\left[R^{a, M}, P_{b}\right]=\delta_{b}^{a} \Theta^{M}{ }_{\alpha} R^{\alpha} \equiv \delta_{b}^{a} T^{N}
$$

Since the other form generators are the result of multiple commutators of the 1-form generators, see eqs. (5.8), (5.9) and (5.11), we may use the Jacobi identities to find the commutator of $P_{a}$ with any of the higher form generators. The result for the 2-form generator $R^{a b}{ }_{M}$ is

$$
\left[R^{a b}{ }_{M}, P_{c}\right]=Z_{M N}\left(\delta_{c}^{a} R^{b, N}-\delta_{c}^{b} R^{a, N}\right),
$$


where

$$
Z_{M N}=d_{M P Q} \Theta_{\alpha}^{P}\left(D^{\alpha}\right)_{N}{ }^{Q}=d_{M P Q}\left(X^{P}\right)_{N}{ }^{Q}
$$

and $X$ is defined by

$$
\left(X^{P}\right)_{M}^{N} \equiv \Theta_{\alpha}^{P}\left(D^{\alpha}\right)_{M}^{N}
$$

In deriving eq. (5.15) we have used the identity $d_{M N P} d^{M N Q}=\delta_{P}^{Q}$. Proceeding in the same way we find the commutators of the 3 and 4 -form generators with $P_{a}$, which are given by

$$
\left[R^{a b c, \alpha}, P_{d}\right]=-\frac{3}{k}\left(D^{\alpha}\right)_{M}{ }^{N}\left(X^{M}\right)_{N}{ }^{P} \delta_{d}^{[a} R^{b c]}{ }_{P}
$$

and

$$
\left[R^{a b c d}{ }_{M N}, P_{e}\right]=-4 Z_{P[M}\left(D_{\alpha}\right)_{N]}^{P} \delta_{e}^{[a} R^{b c d], \alpha}
$$

There are a number of relations that are implied by the Jacobi identities of the generators. For example the Jacobi identity involving $R^{a b}{ }_{M}, P_{c}$ and $P_{d}$ and the relations $\left[P_{c}, P_{d}\right]=0$ implies that

$$
Z_{M N} \Theta^{N}{ }_{\alpha}=0
$$

Writing $T^{N}$ in terms of the commutator of eq. (5.14) and using the Jacobi identities we find that

$$
\left[T^{M}, T^{N}\right]=f^{M N} T^{P}
$$

where the structure constants of the resulting algebra are given by

$$
f_{P}^{M N}=\Theta_{\alpha}^{[M}\left(D^{\alpha}\right)_{P}{ }^{N]}=\left(X^{[M}\right)_{P}^{N]}
$$

which clearly must satisfy the corresponding Jacobi identity when projected onto $\Theta^{M}{ }_{\alpha}$, thus placing more constraints on $\Theta^{M}$. As we will shortly see the sub-algebra of $E_{6}$ that the $T^{N}$ generate will be the gauged, or local, algebra of the massive supergravity.

The constraints on $\Theta^{M}{ }_{\alpha}$ and $Z_{M N}$ we find agree with those found using supersymmetry of reference [20]. Clearly, there are more Jacobi identities and these should lead to all the constraints of reference [20]. In particular, $Z_{M N}$ in eq. (5.16) has to be antisymmetric, thus belonging to the $\overline{\mathbf{3 5 1}}$ of $E_{6}$. The object $\Theta$, which is often called the embedding tensor, belongs to the $\overline{\mathbf{2 7}} \otimes \mathbf{7 8}=\overline{\mathbf{2 7}} \oplus \overline{\mathbf{3 5 1}} \oplus \overline{\mathbf{1 7 2 8}}$, and satisfies constraints that project out the $\overline{\mathbf{2 7}}$ and the $\overline{\mathbf{1 7 2 8}}$, so that only the $\overline{\mathbf{3 5 1}}$ representation survives. Thus the constraints found from supersymmetry and the ones that follow from demanding the consistency of the above algebra are the same and the existence of one implies the existence of the other. 
The reader may be wondering how the above commutators involving $P_{a}$ can be incorporated into a larger algebra involving $E_{11}$. In a subsequent publication [59] we will show how by starting from the non-linear realisation of $E_{11} \otimes_{s} l_{1}$ one can recover the above commutators. However, the $E_{11} \otimes_{s} l_{1}$ formulation resolves certain difficulties one encounters with the above commutators which are really only valid when projected by the deformations being considered. Nonetheless, the above commutators serve the purpose in that they provide a flavour of the underlying derivation of the equations required to find the dynamics, as will become clear in the following.

We will now show that the non-linear realisation of the algebra of the equations above implies the dynamics of the form fields and we will find that the algebra generated by the $T^{N}$ is the gauge algebra and $\Theta^{N}{ }_{\alpha}$ is the mass deformation parameter. In what follows we will only consider the sector of the theory involving gauge fields and scalars, thus neglecting gravity and higher-rank fields. We define the group element to be

$$
g=\exp \left(x^{a} P_{a}\right) g_{A} g_{\phi}
$$

where

$$
g_{A}=e^{A_{a b c, \alpha} R^{a b c, \alpha}} e^{A_{a b}{ }^{M} R^{a b}{ }_{M}} e^{A_{a, M} R^{a, M}}
$$

and

$$
g_{\phi}=e^{\phi_{\alpha} R^{\alpha}}
$$

We demand that the theory is invariant under

$$
g \rightarrow g_{0} g h \quad
$$

where $g_{0}$ is a rigid element from the whole group and $h$ is a local transformation that is generated by elements of the Cartan involution invariant sub-algebra. We calculate the Maurer-Cartan form

$$
\mathcal{V}=g^{-1} d g
$$

which transforms as

$$
\mathcal{V} \rightarrow h^{-1} \mathcal{V} h+h^{-1} d h .
$$

The Cartan forms are invariant under the rigid transformations, but do transform under the local transformations of equation (5.28) and as a result are usually used to construct the dynamics. 
The Cartan form which is the coefficient of the $E_{6}$ generators is used to construct the dynamics of the scalars. For the above algebra this is given by

$$
g_{\phi}^{-1} \partial_{a} g_{\phi}-g_{\phi}^{-1} A_{a, M} \Theta^{M}{ }_{\alpha} R^{\alpha} g_{\phi} \equiv g_{\phi}^{-1} \partial_{a} g_{\phi}-g_{\phi}^{-1} A_{a, M} T^{M} g_{\phi} .
$$

This expression tells us that the theory is gauged with gauge fields $A_{a, M} \Theta^{M}{ }_{\alpha}$ and that some of the scalars are charged under this gauge group. The gauged vector fields are formed by using $\Theta^{M}{ }_{\alpha}$ to project the vectors of the massless theory which belong to the $\overline{\mathbf{2 7}}$ of $E_{6}$ to a subgroup of $E_{6}$.

The term in the Cartan form proportional to the one-form generator $R^{a, M}$ is given by

$$
\partial_{a} A_{b, M}-\frac{1}{2} A_{a, N} \Theta_{\alpha}^{N}\left(D^{\alpha}\right)_{M}^{P} A_{b, P}-2 Z_{M N} A_{a b}{ }^{N} .
$$

As discussed elsewhere [23], one must combine $E_{11}$ with the conformal group and consider the closure of both groups. On doing this one finds that the object which transforms covariantly under both groups is that given above with the $a$ and $b$ indices anti-symmetrised, that is

$$
F_{a b, M}=\partial_{[a} A_{b], M}-\frac{1}{2} A_{[a, N} \Theta^{N}{ }_{\alpha}\left(D^{\alpha}\right)_{M}{ }^{P} A_{b], P}-2 Z_{M N} A_{a b}{ }^{N} .
$$

This is the object that is used to construct the dynamics. We see that the first two terms reproduce the correct non-abelian term corresponding to the gauged group as it contains a term bilinear in $A_{a, P}$ with a coefficient that is the structure constant for the gauge group generated by $T^{M}$, see eq. (5.22). This expression also contains a 2 -form projected by $Z_{M N}$ and therefore possesses a gauge invariance under which the vector transforms as

$$
\delta A_{a, M}=4 Z_{M N} \Lambda_{a}{ }^{N}
$$

where $\Lambda_{a}{ }^{M}$ is the gauge parameter associated to the 2 -form. All these results precisely reproduce those of $[20]$.

The vectors that do not form the adjoint of the gauge group are gauged away using eq. (5.32), and the corresponding 2-forms which are not gauge invariant receive a mass by means of the Stueckelberg mechanism. These fields have to satisfy self-duality conditions in order to guarantee that the correct number of degrees of freedom are propagating.

Summarising, if $\Theta=0$, there is no gauging and the 2-forms do not appear in eq. (5.30). This means that all the 2-forms can be dualised to vectors, as we are in five dimensions, and this reproduces the well-known supergravity results for the massless theory. In the 
gauged theory, that is if $\Theta \neq 0$, the vectors that do not belong to the adjoint of the gauge group can be gauged away, so that their correct description is in terms of 2 -forms. This corresponds to the fact that in gauged maximal five dimensional supergravity the vectors of the abelian theory that are not in the adjoint of the gauge group are dualised to 2-forms. It would be instructive to complete the above non-linear realisation to find the dynamics of all the forms.

Although we have carried out the above derivation for the five dimensional case, the calculation will be very similar in any other dimension. Equations (5.2), (5.3), (5.5), (5.6) and (5.7) just reflect the representations of the internal symmetry group that the form generators belong to and equations (5.8), (5.9) and (5.11) specify how all the form generators are contained in multiple commutators of the 1-form generators. The deformation is introduced by the commutator of the 1 -form generator with the spacetime translations $P_{a}$ and it specifies the gauge group. The commutator with the higher form generators with $P_{a}$ are then specified by the Jacobi identities. This general pattern will be found in all dimensions and as a result will the analogous dynamical results.

In the past, the construction of massive supergravities has been carried out by seeing what deformations the supersymmetry of the massless theory allows. However, as we have shown $E_{11}$ provides a systematic and complete construction of all such theories where all the fields appear together with their magnetic duals. As such, seen from the $E_{11}$ viewpoint the massive theories appear naturally and automatically as a result of the underlying algebra. All the field equations are first order duality conditions. If $\Theta=0$, these duality conditions can be used to express the 2-forms in terms of the vectors, while if $\Theta \neq 0$ the vectors that do not belong to the adjoint of the gauge group can be gauged away using eq. (5.32) and the duality condition becomes a massive self-duality equation for the 2-forms. This result naturally generalises to any dimension, and therefore $E_{11}$ encodes the field content and the equations of the various supergravity theories.

\section{Conclusions}

In this paper we have shown that all maximal supergravity theories in any dimension above two arise from dimensional reduction of the 11-dimensional $E_{11}$ non-linear realisation. In particular, we have determined all the forms, that is gauge fields with totally antisymmetric indices, that arise from dimensional reduction of the 11-dimensional gauge fields described 
in the $E_{11}$ non-linear realisation. The results are summarised in Table 5 . We have also shown that the gauging of five-dimensional maximal supergravity arises from the non-linear realisation. This result can easily be generalised to any dimension.

The $D-1$ forms have field strengths that are dual to masses, and their classification gives all the possible massive deformations of maximal supergravities in any dimension. Our results are in complete agreement with the classification of gauged supergravities in nine dimensions [16] and in any dimension from seven to three [18, 19, 20, 21, 22] that has been found using supersymmetry. The 8-dimensional case is an exception, because although a classification of gauged supergravities has been provided using the Bianchi classification of group manifolds [17], these results are not formulated in terms of representations of $S L(3, \mathbb{R}) \times S L(2, \mathbb{R}) . E_{11}$ predicts a mass parameter in the $(\overline{\mathbf{6}}, \mathbf{2}) \oplus(\mathbf{3}, \mathbf{2})$, and it would be of interest to check this prediction in detail in this case. Finally, the $D$ forms that we find have not been derived from an alternative approach, with the exception of the 10-dimensional case $[35,38]$. We would like to stress that the derivation of the forms from $E_{11}$ is a very straightforward exercise just involving the algebra.

Hence, taken together with other results, large numbers of the fields that occur in the $E_{11}$ non-linear realisation and are beyond the supergravity approximation have been found to have a physical meaning. Some of the fields for which a physical meaning has been found are buried deep within the Kac-Moody algebra and are associated with rather negative root length squared, and some with multiplicities greater than one. Apart from the usual fields associated with the propagating degrees of freedom of the maximal supergravity theory, $E_{11}$ contains their standard magnetic duals as well as fields corresponding to all possible dual formulations of the physical degrees of freedom. It also contains fields that result in forms with one less dimension than the space-time they are in, leading to all known massive supergravities, as well as forms that have the same rank as the number of dimensions of space-time and are associated with space-filling branes.

While a physical meaning has not been found for all fields in the $E_{11}$ non-linear realisation it would seem very likely that they do possess such an interpretation and they do not lead to more propagating degrees of freedom beyond those of the maximal supergravity theory being considered. Thus, even the reader of a sceptical disposition might well conceed that $E_{11}$ is very likely to be a symmetry of the low energy effective actions of string theory and $\mathrm{M}$ theory, as indeed originally conjectured [23].

Further propagating degrees of freedom might arise from the way spacetime is incor- 
porated in the theory. As suggested in a recent paper [60], the additional coordinates that enter when spacetime translations are enlarged to be part of an $E_{11}$ multiplet may lead to propagating states in addition to all the fields discussed above. This point is under study.

The results of this paper naturally lead to a number of new investigations. It would be interesting to check that the maximal supersymmetry algebras below ten dimensions can be extended in order to include the $D-1$ and spacetime filling forms predicted by $E_{11}$. In particular, the ten-dimensional results of references $[35,38]$ can be extended determining all the forms that are compatible with the closure of the supersymmetry algebra below ten dimensions. This would be an additional confirmation that the predictions of $E_{11}$ are correct. It would also be interesting to see the constraints for the corresponding branes. In particular, the charges of the spacetime-filling branes in IIB are constrained to belong to a non-linear doublet out of the quadruplet of 10-forms [50,51]. A similar analysis in lower dimensions should give rise to constraints for the charges associated to the spacetime-filling forms that we have found.

In would also be interesting to use $E_{11}$ to find the 3 -forms that occur in three dimensions. In order to do this, one should find the multiplicity of the solutions of eq. (2.10) using eq. (2.21) with $q_{8} \geq 1$ that we have found. The number of such solutions is finite, and once the multiplicities are obtained, the resulting 3 -forms group in representations of $E_{8}$. It would then be interesting to match this with the result one could get using supersymmetry. One could also repeat the same analysis in two dimensions, studying the 1-forms and 2-forms that arise. In two dimensions, the field strengths of the 1-forms are dual to masses, and from the analysis carried out in this paper it is evident that there are an infinite number of such forms, corresponding to an infinite number of deformations. This is consistent with the expected infinite-dimensional $E_{9}$ symmetry in two dimensions.

There exists a formulation of M-theory based on the Kac-Moody algebra $E_{10}$ [61]; although $E_{10}$ is a subalgebra of $E_{11}$, the way of introducing spacetime and the resulting dynamics are different to the $E_{11}$ formulation used in this paper. Examining the $E_{10}$ tables in [44] one can see that the corresponding fields to those of Tables 1 and 2 are also present, while all the fields in Table 3 are missing. Thus one would expect the $E_{10}$ formulation to also be able to recover the gauged supergravity theories along the lines of this paper, while the spacetime-filling forms are absent.

The fact that the 9-form of the IIA theory arises from the 11-dimensional field $\hat{A}_{10,1,1}$ will provide the correct framework to understand how the D8-branes are uplifted to 11 dimen- 
sions. Similar considerations apply to the 11-dimensional origin of the non-perturbative $E_{8} \times E_{8}$ heterotic theory, which is conjectured to be dual to M-theory compactified on $S^{1} / \mathbb{Z}_{2}[62]$.

Another phenomenon in lower dimensions that has implications for how we think about M theory was the use of U-duality transformations to find the point particle and string charges multiplets in say three dimensions [63,64]. These multiplets generically contain more charges than occur in the supersymmetry algebra and some of them have a rather exotic index structure. From the $E_{11}$ view point the brane charges are contained in the $E_{11}$ multiplet whose first component is the space-time translations, this is just the fundamental representation of $E_{11}$ which is associated with the node labelled one [65]. The dimensional reduction of this single multiplet to lower dimensions has to lead to charge multiplets which are in complete agreement [66] with those found in references [63, 64]. However, this also provides an eleven dimensional origin for these charges.

The classification of the gauged supergravities using $E_{11}$ given in this paper is similar in that probes of $\mathrm{M}$ theory in lower dimensions have found to be precisely accounted for by $E_{11}$. However, the work on deformations [18, 19, 20, 21, 22] provides a more sophisticated probe in that it relies to a lesser extent on the properties of the internal symmetry group and uses the detailed structure of supergravity theories. The different lower dimensional probes can be seen to reveal different aspects of the underlying $E_{11}$ symmetry, torus dimensional reduction being just the first to be found. Another lower dimensional probe is that of reference [67] which involves del Pezzo surfaces, and it is interesting to note that some of the same internal symmetry multiplets arise. As such, it would be interesting to see how these results emerge from $E_{11}$.

\section{Note added}

While this work was in the final stages of being written up, we learnt from B. de Wit that he and H. Samtleben and H. Nicolai have further extended the analysis of gauged supergravities of Ref. [31] to include some higher tensor gauge fields and their results appear to be in agreement with those found in this paper [68], in particular they have recovered from their viewpoint some, but not all, of the entries shown in Table 5. 


\section{Acknowledgments}

We are grateful to H. Samtleben for discussions about gauged supergravities and to B. de Wit for drawing our attention to Ref. [67]. P.W. would like to thank A. Pressley for discussions on Kac-Moody algebras. F.R. would like to thank E. Bergshoeff for discussions at an early stage of this work. We thank the Galileo Galilei Institute for Theoretical Physics for the hospitality and INFN for partial support during the final stages of writing up this work. The research of P.W. was supported by a PPARC senior fellowship PPA/Y/S/2002/001/44. The work of both authors is also supported by a PPARC rolling grant PP/C5071745/1 and the EU Marie Curie, research training network grant HPRN-CT-2000-00122.

\section{References}

[1] I. C. G. Campbell and P. C. West, "N=2 D = 10 Nonchiral Supergravity And Its Spontaneous Compactification," Nucl. Phys. B 243 (1984) 112; F. Giani and M. Pernici, "N=2 Supergravity In Ten-Dimensions," Phys. Rev. D 30 (1984) 325; M. Huq and M. A. Namazie, "Kaluza-Klein Supergravity In Ten-Dimensions," Class. Quant. Grav. 2 (1985) 293 [Erratum-ibid. 2 (1985) 597].

[2] J. H. Schwarz and P. C. West, "Symmetries And Transformations Of Chiral N=2 D = 10 Supergravity," Phys. Lett. B 126 (1983) 301.

[3] P. S. Howe and P. C. West, "The Complete N=2, D = 10 Supergravity," Nucl. Phys. B 238 (1984) 181.

[4] J. H. Schwarz, "Covariant Field Equations Of Chiral N=2 D = 10 Supergravity," Nucl. Phys. B 226 (1983) 269.

[5] E. Cremmer, B. Julia and J. Scherk, "Supergravity theory in 11 dimensions," Phys. Lett. B 76 (1978) 409.

[6] E. Cremmer and B. Julia, "The $N=8$ Supergravity Theory. 1. The Lagrangian," Phys. Lett. B 80 (1978) 48; "The SO(8) Supergravity," Nucl. Phys. B 159 (1979) 141. 
[7] B. Julia, "Group Disintegrations," in Superspace and Supergravity, Eds. S.W. Hawking and M. Rocek (Cambridge Univ. Press, 1981).

[8] M. Henneaux and C. Teitelboim, "Quantization of topological mass in the presence of a magnetic pole," Phys. Rev. Lett. 56 (1986) 689.

[9] A. Sen, "Electric magnetic duality in string theory," Nucl. Phys. B 404 (1993) 109 [arXiv:hep-th/9207053].

[10] A. Font, L. E. Ibanez, D. Lust and F. Quevedo, "Strong - weak coupling duality and nonperturbative effects in string theory," Phys. Lett. B 249 (1990) 35.

[11] C. M. Hull and P. K. Townsend, "Unity of superstring dualities," Nucl. Phys. B 438 (1995) 109 [arXiv:hep-th/9410167].

[12] B. de Wit and H. Nicolai, "N=8 Supergravity With Local SO(8) X SU(8) Invariance," Phys. Lett. B 108 (1982) 285. “N=8 Supergravity,” Nucl. Phys. B 208 (1982) 323.

[13] L. J. Romans, "Massive N=2a Supergravity In Ten-Dimensions," Phys. Lett. B 169 (1986) 374.

[14] M. Pernici, K. Pilch and P. van Nieuwenhuizen, "Gauged N=8 D=5 Supergravity," Nucl. Phys. B 259 (1985) 460; M. Gunaydin, L. J. Romans and N. P. Warner, "Gauged N=8 Supergravity In Five-Dimensions," Phys. Lett. B 154 (1985) 268.

[15] E. Cremmer, "Supergravities In 5 Dimensions," in Superspace and Supergravity, Eds. S.W. Hawking and M. Rocek (Cambridge Univ. Press, 1981).

[16] E. Bergshoeff, T. de Wit, U. Gran, R. Linares and D. Roest, "(Non-)Abelian gauged supergravities in nine dimensions," JHEP 0210 (2002) 061 [arXiv:hep-th/0209205].

[17] E. Bergshoeff, U. Gran, R. Linares, M. Nielsen, T. Ortin and D. Roest, "The Bianchi classification of maximal D = 8 gauged supergravities," Class. Quant. Grav. 20 (2003) 3997 [arXiv:hep-th/0306179].

[18] H. Samtleben and M. Weidner, "The maximal D = 7 supergravities," Nucl. Phys. B 725 (2005) 383 [arXiv:hep-th/0506237].

[19] B. de Wit, H. Samtleben and M. Trigiante, "On Lagrangians and gaugings of maximal supergravities," Nucl. Phys. B 655 (2003) 93 [arXiv:hep-th/0212239].

[20] B. de Wit, H. Samtleben and M. Trigiante, "The maximal D = 5 supergravities," Nucl. Phys. B 716 (2005) 215 [arXiv:hep-th/0412173]. 
[21] B. de Wit, H. Samtleben and M. Trigiante, "Magnetic charges in local field theory," JHEP 0509 (2005) 016 [arXiv:hep-th/0507289].

[22] H. Nicolai and H. Samtleben, "Maximal gauged supergravity in three dimensions," Phys. Rev. Lett. 86 (2001) 1686 [arXiv:hep-th/0010076].

[23] P. C. West, "E(11) and M theory," Class. Quant. Grav. 18 (2001) 4443 [arXiv:hepth/0104081].

[24] P. C. West, "Hidden superconformal symmetry in M theory," JHEP 0008 (2000) 007 [arXiv:hep-th/0005270].

[25] I. Schnakenburg and P. C. West, "Kac-Moody symmetries of IIB supergravity," Phys. Lett. B 517 (2001) 421 [arXiv:hep-th/0107181].

[26] A. Kleinschmidt, I. Schnakenburg and P. West, "Very-extended Kac-Moody algebras and their interpretation at low levels," Class. Quant. Grav. 21 (2004) 2493 [arXiv:hepth/0309198].

[27] J. Polchinski and E. Witten, "Evidence for Heterotic - Type I String Duality," Nucl. Phys. B 460 (1996) 525 [arXiv:hep-th/9510169].

[28] E. Bergshoeff, M. de Roo, M. B. Green, G. Papadopoulos and P. K. Townsend, "Duality of Type II 7-branes and 8-branes," Nucl. Phys. B 470 (1996) 113 [arXiv:hepth/9601150].

[29] I. Schnakenburg and P. C. West, "Massive IIA supergravity as a non-linear realisation," Phys. Lett. B 540 (2002) 137 [arXiv:hep-th/0204207].

[30] E. Bergshoeff, R. Kallosh, T. Ortin, D. Roest and A. Van Proeyen, "New formulations of D = 10 supersymmetry and D8 - O8 domain walls," Class. Quant. Grav. 18 (2001) 3359 [arXiv:hep-th/0103233].

[31] B. de Wit and H. Samtleben, "Gauged maximal supergravities and hierarchies of nonabelian vector-tensor systems," Fortsch. Phys. 53 (2005) 442 [arXiv:hep-th/0501243].

[32] E. Cremmer, B. Julia, H. Lu and C. N. Pope, "Dualisation of dualities. II: Twisted selfduality of doubled fields and superdualities," Nucl. Phys. B 535 (1998) 242 [arXiv:hepth/9806106].

[33] G. Dall'Agata, K. Lechner and M. Tonin, "D = 10, N = IIB supergravity: Lorentzinvariant actions and duality," JHEP 9807 (1998) 017 [arXiv:hep-th/9806140]. 
[34] P. Meessen and T. Ortin, "An $\mathrm{Sl}(2, \mathrm{Z})$ multiplet of nine-dimensional type II supergravity theories," Nucl. Phys. B 541 (1999) 195 [arXiv:hep-th/9806120].

[35] E. A. Bergshoeff, M. de Roo, S. F. Kerstan and F. Riccioni, "IIB supergravity revisited," JHEP 0508 (2005) 098 [arXiv:hep-th/0506013].

[36] P. West, "E(11), ten forms and supergravity," JHEP 0603 (2006) 072 [arXiv:hepth/0511153].

[37] F. Riccioni, "Spacetime-filling branes in ten and nine dimensions," Nucl. Phys. B 711 (2005) 231 [arXiv:hep-th/0410185].

[38] E. A. Bergshoeff, M. de Roo, S. F. Kerstan, T. Ortin and F. Riccioni, "IIA ten-forms and the gauge algebras of maximal supergravity theories," JHEP 0607 (2006) 018 [arXiv:hep-th/0602280].

[39] F. Riccioni and P. West, "Dual fields and E(11)," Phys. Lett. B 645 (2007) 286 [arXiv:hep-th/0612001].

[40] M. R. Gaberdiel, D. I. Olive and P. C. West, "A class of Lorentzian Kac-Moody algebras," Nucl. Phys. B 645 (2002) 403 [arXiv:hep-th/0205068].

[41] T. Damour, M. Henneaux and H. Nicolai, "E(10) and a 'small tension expansion' of M theory," Phys. Rev. Lett. 89 (2002) 221601 [arXiv:hep-th/0207267].

[42] P. West, "Very extended E(8) and A(8) at low levels, gravity and supergravity," Class. Quant. Grav. 20 (2003) 2393 [arXiv:hep-th/0212291].

[43] For a review, see: V. G. Kac, "Infinite dimensional Lie algebras," Cambridge, UK: Univ. Pr. (1990) 400 p.

[44] H. Nicolai and T. Fischbacher, "Low level representations for E(10) and E(11)," arXiv:hep-th/0301017.

[45] V. I. Ogievetsky, "Infinite-dimensional algebra of general covariance group as the closure of finite-dimensional algebras of conformal and linear groups," Lett. Nuovo Cim. 8 (1973) 988.

[46] A. B. Borisov and V. I. Ogievetsky, "Theory of dynamical affine and conformal symmetries as gravity theory of the gravitational field," Theor. Math. Phys. 21 (1975) 1179 [Teor. Mat. Fiz. 21 (1974) 329].

[47] R. Slansky, "Group Theory For Unified Model Building," Phys. Rept. 79 (1981) 1. 
[48] P. West, "The IIA, IIB and eleven dimensional theories and their common E(11) origin," Nucl. Phys. B 693 (2004) 76 [arXiv:hep-th/0402140].

[49] M. Gunaydin and N. Marcus, "The Spectrum Of The $\mathrm{S}^{* *} 5$ Compactification Of The Chiral $N=2, D=10$ Supergravity And The Unitary Supermultiplets Of U(2, 2/4)," Class. Quant. Grav. 2 (1985) L11; H. J. Kim, L. J. Romans and P. van Nieuwenhuizen, "The Mass Spectrum Of Chiral N=2 D=10 Supergravity On S**5," Phys. Rev. D 32 (1985) 389.

[50] E. A. Bergshoeff, M. de Roo, S. F. Kerstan, T. Ortin and F. Riccioni, "IIB ninebranes," JHEP 0606 (2006) 006 [arXiv:hep-th/0601128].

[51] E. A. Bergshoeff, M. de Roo, S. F. Kerstan, T. Ortin and F. Riccioni, "SL(2,R)invariant IIB brane actions," JHEP 0702 (2007) 007 [arXiv:hep-th/0611036].

[52] B. L. Julia, "Dualities in the classical supergravity limits: Dualisations, dualities and a detour via 4k+2 dimensions," arXiv:hep-th/9805083.

[53] P. S. Howe, N. D. Lambert and P. C. West, "A new massive type IIA supergravity from compactification," Phys. Lett. B 416 (1998) 303 [arXiv:hep-th/9707139].

[54] A. Salam and E. Sezgin, "D = 8 Supergravity," Nucl. Phys. B 258 (1985) 284.

[55] E. Sezgin and A. Salam, "Maximal Extended Supergravity Theory In SevenDimensions," Phys. Lett. B 118 (1982) 359.

[56] M. Pernici, K. Pilch and P. van Nieuwenhuizen, "Gauged Maximally Extended Supergravity In Seven-Dimensions," Phys. Lett. B 143 (1984) 103.

[57] Y. Tanii, "N=8 Supergravity In Six-Dimensions," Phys. Lett. B 145 (1984) 197.

[58] N. Marcus and J. H. Schwarz, "Three-Dimensional Supergravity Theories," Nucl. Phys. B 228 (1983) 145.

[59] F. Riccioni and P. West, work in progress.

[60] P. West, " $E_{11}$ and Higher Spin Theories," arXiv:hep-th/0701026.

[61] T. Damour, M. Henneaux and H. Nicolai, "E(10) and a 'small tension expansion' of M theory," Phys. Rev. Lett. 89 (2002) 221601 [arXiv:hep-th/0207267].

[62] P. Horava and E. Witten, "Heterotic and type I string dynamics from eleven dimensions," Nucl. Phys. B 460 (1996) 506 [arXiv:hep-th/9510209]. 
[63] S. Elitzur, A. Giveon, D. Kutasov and E. Rabinovici, "Algebraic aspects of matrix theory on T**d," Nucl. Phys. B 509 (1998) 122 [arXiv:hep-th/9707217].

[64] N. A. Obers, B. Pioline and E. Rabinovici, "M-theory and U-duality on $\mathrm{T}^{* *} \mathrm{~d}$ with gauge backgrounds," Nucl. Phys. B 525 (1998) 163 [arXiv:hep-th/9712084]; N. A. Obers and B. Pioline, "U-duality and M-theory," Phys. Rept. 318 (1999) 113 [arXiv:hep-th/9809039]; N. A. Obers and B. Pioline, "U-duality and M-theory, an algebraic approach," arXiv:hep-th/9812139.

[65] P. West, "E(11), SL(32) and central charges," Phys. Lett. B 575 (2003) 333 [arXiv:hep-th/0307098].

[66] P. West, "E(11) origin of brane charges and U-duality multiplets," JHEP 0408 (2004) 052 [arXiv:hep-th/0406150].

[67] A. Iqbal, A. Neitzke and C. Vafa, "A mysterious duality," Adv. Theor. Math. Phys. 5 (2002) 769 [arXiv:hep-th/0111068].

[68] B. de Wit, H. Nicolai and H. Samtleben, work in progress. 


\begin{tabular}{|c|c|c|c|c|c|c|c|c|c|c|c|}
\hline D & G & 1-forms & 2 -forms & 3 -forms & 4 -forms & 5-forms & 6-forms & 7-forms & 8-forms & 9-forms & 10-forms \\
\hline $10 \mathrm{~A}$ & $\mathbb{R}^{+}$ & 1 & 1 & 1 & & 1 & 1 & 1 & 1 & 1 & $\begin{array}{l}1 \\
1\end{array}$ \\
\hline 10B & $S L(2, \mathbb{R})$ & & 2 & & 1 & & 2 & & 3 & & $\begin{array}{l}4 \\
2 \\
\end{array}$ \\
\hline 9 & $S L(2, \mathbb{R}) \times \mathbb{R}^{+}$ & $\begin{array}{l}2 \\
1 \\
\end{array}$ & 2 & 1 & 1 & 2 & $\begin{array}{l}2 \\
1 \\
\end{array}$ & $\begin{array}{l}3 \\
1 \\
1\end{array}$ & $\begin{array}{l}3 \\
2 \\
\end{array}$ & $\begin{array}{l}4 \\
2 \\
2 \\
\end{array}$ & \\
\hline 8 & $S L(3, \mathbb{R}) \times S L(2, \mathbb{R})$ & $(\overline{3}, 2)$ & $(3,1)$ & $(1,2)$ & $(\overline{3}, 1)$ & $(3,2)$ & $\begin{array}{l}(8,1) \\
(1,3)\end{array}$ & $\begin{array}{l}(6,2) \\
(\overline{3}, 2)\end{array}$ & $\begin{array}{c}(15,1) \\
(3,3) \\
(3,1) \\
(3,1) \\
\end{array}$ & & \\
\hline 7 & $S L(5, \mathbb{R})$ & $\overline{10}$ & 5 & $\overline{5}$ & 10 & 24 & $\begin{array}{l}\overline{40} \\
\overline{15}\end{array}$ & $\begin{array}{c}70 \\
45 \\
5 \\
\end{array}$ & & & \\
\hline 6 & $S O(5,5)$ & 16 & 10 & $\overline{16}$ & 45 & 144 & $\begin{array}{c}\frac{320}{126} \\
10\end{array}$ & & & & \\
\hline 5 & $E_{6(+6)}$ & 27 & $\overline{27}$ & 78 & 351 & $\begin{array}{c}\overline{1728} \\
\overline{27}\end{array}$ & & & & & \\
\hline 4 & $E_{7(+7)}$ & 56 & 133 & 912 & $\begin{array}{c}8645 \\
133 \\
\end{array}$ & & & & & & \\
\hline 3 & $E_{8(+8)}$ & 248 & $\begin{array}{c}3875 \\
1\end{array}$ & ? & & & & & & & \\
\hline
\end{tabular}

Table 5: Table giving the representations of the symmetry group $G$ of all the forms of maximal supergravities in any dimension. The $D-2$-forms dual to the scalars always belong to the adjoint representation. The scalars, parametrising the coset $\mathrm{G} / \mathrm{H}$, are not included in the table. The 3 -forms in 3 dimensions are inside the $\mathbf{2 4 8} \oplus \mathbf{2 4 8} \oplus \mathbf{3 8 7 5} \oplus \mathbf{3 0 3 8 0} \oplus \mathbf{1 4 7 2 5 0} \oplus \mathbf{7 7 9 2 4 7}$ of $E_{8}$. 